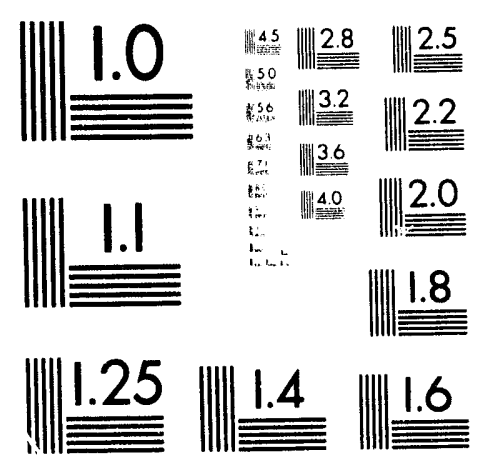



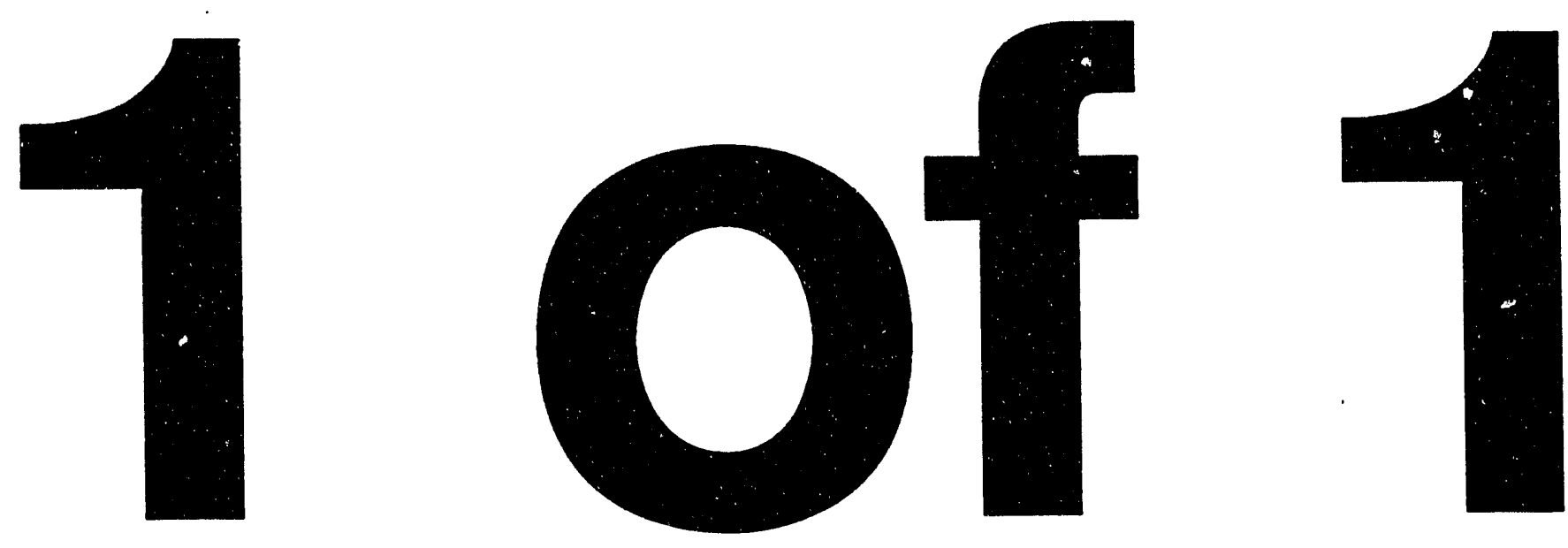


\title{
Three-Nucleon Force
}

\author{
and
}

\section{the $\Delta$-Mechanism for Pion Production and Pion Absorption}

\author{
M. T. Peña \\ Centro de Fisica .Vuclear da Liniversidade de Lisboa, P-1699 Lisboa Codex, Portugal \\ and \\ Continuous Electron Beam Accelerator Facility, Newport News, Virginia 23606. L.S.A. \\ P. U. Sauer \\ Institut für Theoretische Physik, Universität Hannover, D-3000 Hannover 1, Germany
}

A. Stadler

Institut für Theoretische Physik, Universität Hannover, D-3000 Hannover 1. Germany and

Department of Physics, College of William and Mary, Williamsburg, Virginia 23185, U.S.A."

G. Korteme yer

Institut für Theoretische Physik, Universität Hannover, D-3000 Hannover 1, Germany

(May 18, 1993)

\begin{abstract}
The description of the three-nucleon system in terms of nucleon and $\Delta$ degrees of freedom is extended to allow for explicit pion production (absorption) from single dynamic $\Delta$ de-excitation (excitation) processes. This mechanism yields an energy dependent effective three-body hamiltonean. The Faddeev equations for the trinucleon bound state are solved with a force model that has already been tested in the two-nucleon system above pion-production threshold. The binding energy and other bound state properties are calculated. The contribution to the effective three-nucleon force arising from the pionic degrees of freedom is evaluated. The validity of previous coupled-channel calculations with explicit but stable $\Delta$ isobar components in the wavefunction is studied.
\end{abstract}

$21.30 .+\mathrm{y}, 21.10 . \mathrm{Dr}, 21.45+\mathrm{v}, 27.10 .+\mathrm{h}$ 


\section{INTRODUCTION}

Two-nucleon and three-nucleon forces are effective interactions [1]. A truely microscopic description of the nucleus in terms of quantum chromodynamics with quark and gluon degrees of freedom would avoid the notion of two-nucleon and three-nucleon forces altogether. If one views the nucleus in the more traditional hadronic picture as a system of nucleons. isobars. their corresponding antiparticles and mesons, one encounters interactions in form of baryon-meson vertices, but again no two-nucleon and three-nucleon forces. Two-nucleon and three-nucleon forces arise when subnucleonic degrees of freedom are frozen. They are therefore artifacts of theoreticians who choose to work in a Hilbert space with a restricted number of degrees of freedom.

At intermediate energies the pion and $\Delta$-isobar degrees of freedom become active in nuclear reactions. Any efficient description of nuclear phenomena at intermediate energies has to treat the pion and $د$-isobar degrees of freedom explicitly besides the nucleon one. A Hilbert space doing so is illustrated in Fig. 1. It contains - besides the purely nucleonic sector $\mathcal{H}_{,}$- a sector $\mathcal{H}_{\Delta}$ in which one nucleon is replaced by a $\Delta$-isobar and a sector $\mathcal{H}$. in which one pion is added to the nucleons. That extended. though still rather restricted Hilbert space is motivated in Ref. [2]. A force model acting in that Hilbert space is developed in Refs. [2] and [3]. Its hamiltonian is diagrammatically defined in Fig. 2. It is not covariant. Its unlinked one-baryon processes (e) and (f) can fully account for pion-nucleon scattering up to $300 \mathrm{MeV}$ pion lab energy. Those processes are to be parametrized consistently with data. The $\Delta$-isobar of the force model is a bare particle which only by its coupling to pion-nucleon states describes the physical $P_{33}$ resonance. The force model builds up the mechanism for pion production or pion absorption as a two-step process, i.e.,

- by the excitation of a nucleon to or by the deexcitation of a $\Delta$-isobar through the instantaneous transition potential (b) and

- by the subsequent decay of that $د$-isobar into or by its formation from pion-nucleon states through the pion-nucleon- $\Delta$ vertex $(\mathrm{e})$.

The force model is tested in the two-nucleon system above pion-threshold, e.g., in Refs. [4] and [5] for all reactions with at most one pion, coupled by unitarity. It is not tuned yet and therefore fails to account for many observables as any nucleon-nucleon potential would without a proper fit. However, the force model is constructed to account for nuclenn-nucleon scattering below pion-threshold with satisfactory quality. A comparison with other force models of similar structure is given in Ref. [6].

The force model of Fig. 2 is not only meant to account for the two-nucleon system below and above pion threshold. In heavier nuclei it can also provide a microscopic basis for describing reactions at intermediate energies, and the force model has been employed that way [7]. When applied to nuclear structure problems, the force model yields corrections [8] for the picture of the nucleus as a system of nucleons only interacting through two-nucleon forces with each other and through single-nucleon currents with exte:nal probes. E.g., when applied to the trinucleon bound state, it yields corrections to the effective three-nucleon force and to the effective two- and three-nucleon currents $[9,10]$. 


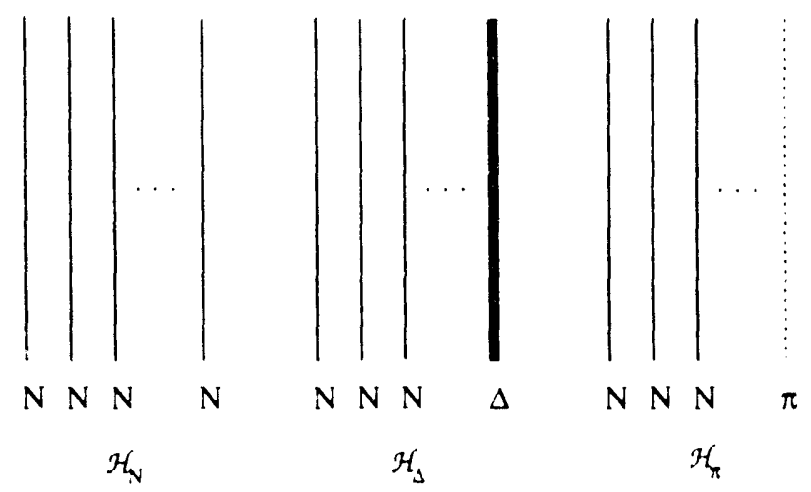

FIG. 1. Hilbert space for a many-nucleon system. Besides the purely nucleonic sector $\mathcal{H}_{N}$ there

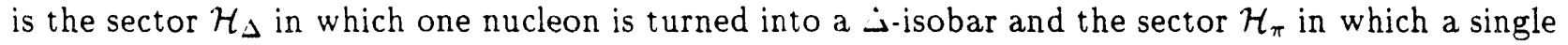
pion is added.

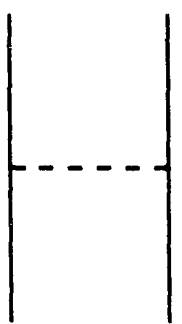

(a)

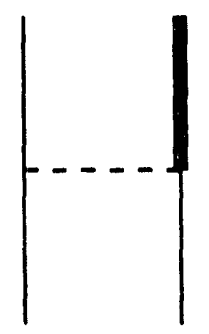

(b)

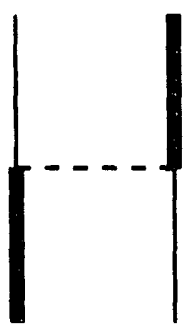

(c)

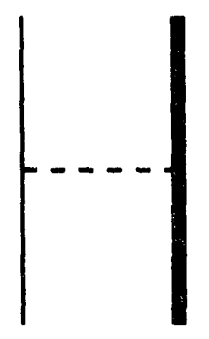

(d)

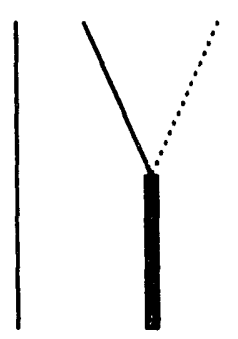

(e)

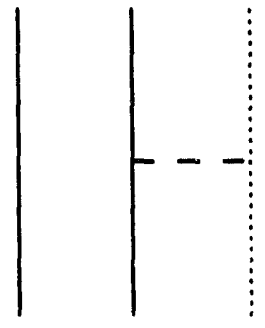

$(f)$

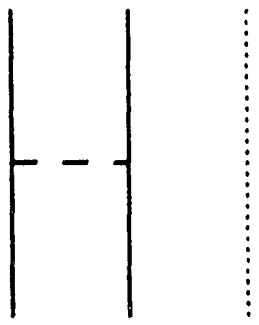

(g)

FIG. 2. Building blocks of the force model with $\Delta$-isobar and pion degrees of freetom. The hermitian adjoint pieces corresponding to the processes (b) and (e) are not shown. The $\Delta$-isobar is a bare particle; process (e) yields the physical $P_{33}$ pion-nucleon resonance by iteration; process (f) stands for the nonresonant pion-nucleon interactions: in generai, it could also have contributions in $P_{33}$; none of those possible $P_{33}$ background contributions is indicated in the following Figs. 3 and 4. The extended force model acts in isospin-triplet partial waves only. In isospin-singlet partial waves the force model is purely nucleonic and reduces to process (a). 
Characteristic examples for contributions to the three-nucleon force are illustrated in Fig. 3.

$$
\mid+1
$$
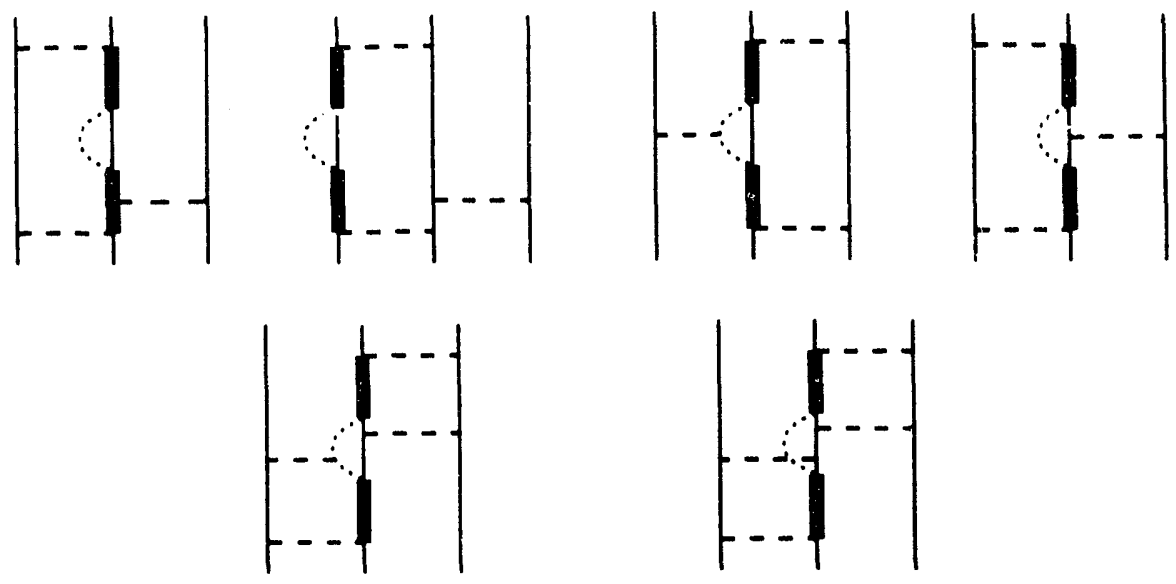

FIG. 3. Examples for contributions to the effective three-nucleon force arising in a three-nucleon system from the force model of Fig. 2. The contributions are irreducible in the purely nucleonic Hilbert sector $\mathcal{H}_{N}$. Selected contributions up to fourth order in two-particle potentials are shown. With respect to the pion-nucleon interaction, possible $P_{33}$ background contributions are not considered in this figure.

It has been argued [11,12] and shown [13] that the contributions arising from the $\Delta$-isobar and the pion degrees of freedom are most important in a full and realistic three-nucleon force. Subject of this paper is the relation between the three-nucleon force and the force model of Fig. 2 with $\Delta$-isobar and pion degrees of freedom. Preliminary discussiuns of that theme have been given in Refs. [14] and [15].

Sect. II recalls the precise definition of the force model of Fig. 2. It applies the force model to the trinucleon bound state. The resulting set of equations is very close to those of a coupled-channel treatment $[9,10]$ for nuclear bound states, in which the $\Delta$-isobar is considered a stable particle with fixed mass. Sect. III describes the actual calculations carried out in this paper for the full force model and for approximated variants of it. The calculations are meant to explore the validity of the corresponding coupled-channel description of nuclear bound states. Sect. IV presents the obtained results. Sect. V discusses conclusions. 


\section{APPLICATION OF THE FORCE MODEL WITH $\triangle$-ISOBAR AND PION DEGREES OF FREEDOM TO THE TRINUCLEON BOUND STATE}

The hamiltonian $H$ of the force model acts in the three sectors of Hilbert space and couples them. The projectors on the two baryonic sectors $\mathcal{H}_{N}$ and $\mathcal{H}_{\Delta}$ are denoted by $P_{N}$ and $P_{\Delta}$. respectively, with the abbreviation $P=P_{N}+P_{\Delta}$, the projector on the sector $\mathcal{H}_{\pi}$ with a pion by $Q_{\mathfrak{c}}$. Thus. $P_{v}+P_{\Delta}+Q=1$. The kinetic part $H_{0}$ of the hamiltonian defines the Hilbert sectors and commutes with the projectors. It consists of the individual baryonic contributions $h_{0}(i), i$ being the baryon label: in the Hilbert sector $\mathcal{H}_{\pi}$ it has the additional pion contribution $h_{0}(\pi)$. It includes rest masses. The mass $m_{\Delta}^{0}$ of the bare $\Delta$-isobar used in the definitions of the Hilbert sector $\mathcal{H}_{\Delta}$ and of the force model is unobservable. The singleparticle momentum of a nucleon, a $\boldsymbol{J}$-isobar and a pion is $\mathbf{k}_{\mathrm{N}}, \mathrm{k}_{\Delta}$ and $\mathbf{k}_{\pi}$. respectiviv. The kinetic energies of the nucleon and of the $\lrcorner$-isobar are taken to be nonrelativistic, i.e., $\Sigma_{N}\left(k_{N}\right)=m_{N}+k_{N}^{2} / 2 m_{v}$ and $\Sigma_{د}\left(k_{\Delta}\right)=m_{\Delta}^{0}+k_{\Delta}^{2} / 2 m_{\Delta}^{0}$, the kinetic energy of the pion to be relativistic, i.e.. $\omega_{-}\left(k_{\pi}\right)=\sqrt{m_{\pi}^{2}+k_{\pi}^{2}}$. The interaction part $H_{1}$ of the hamiltonian is built from instantaneous unretarded potentials. It connects the pionic sector only to the one with a $\triangle$-isobar, thus, $P_{. v} H_{1} Q=Q H_{1} P_{N}=0$. The interaction part of the hamiltonian takes the form

$$
\begin{aligned}
H_{1}= & \left(P_{N}+P_{\Delta}\right) H_{1}\left(P_{N}+P_{\Delta}\right)+ \\
& P_{\Delta} H_{1} Q+Q H_{1} P_{\Delta}+Q H_{1} Q .
\end{aligned}
$$

The Schrödinger equation for the trinucleon bound-state energy $E_{B}$ and for the wave function $\left|\Psi_{B}\right\rangle$

$$
\left[H_{0}+H_{1}\right]\left|\Psi_{B}\right\rangle=E_{B}\left|\Psi_{B}\right\rangle
$$

is projected onto the baryonic and pionic sectors of Hilbert space, i.e.,

$$
\begin{gathered}
{\left[P\left(H_{0}+\delta H_{0}\left(E_{B}\right)\right) P+P\left(H_{1}+\delta H_{1}\left(E_{B}\right)\right) P\right] P\left|\Psi_{B}\right\rangle} \\
=E_{B} P\left|\Psi_{B}\right\rangle, \\
Q\left|\Psi_{B}\right\rangle=\frac{Q}{E_{B}-Q H Q} Q H_{1} P P\left|\Psi_{B}\right\rangle, \\
\left\langle\Psi_{B}|P| \Psi_{B}\right\rangle+\left\langle\Psi_{B}|Q| \Psi_{B}\right\rangle=1 .
\end{gathered}
$$

The triton binding energy $E_{T}$ without rest masses, i e., $E_{T}=E_{B}-3 m_{N}$, and the baryonic components $\left(P_{N}+P_{\Delta}\right)\left|\Psi_{B}\right\rangle$ of the urinucleon wave inction follow from solving the projected equation (2.3a). The pionic component $Q\left|\Psi_{B}\right\rangle$ of the wave function is obtained from its $\Delta$ component $P_{\Delta}\left|\Psi_{B}\right\rangle$ according to Eq. (2.3b) by quadrature. The projected equation (2.3a) contains the energy-dependent parts $\delta H_{0}\left(E_{B}\right)$ and $\delta H_{1}\left(E_{B}\right)$, i.e., 


$$
\begin{aligned}
& P \delta H_{0}(z) P=\left[P H_{1} Q \frac{Q}{z-Q H Q} Q H_{1} P\right]_{\text {disconnecied }}, \\
& P \delta H_{1}(z) P=\left[P H_{1} Q \frac{Q}{z-Q H Q} Q H_{1} P\right]_{\text {connecied }} .
\end{aligned}
$$

The disconnected part $\delta H_{0}(z)$ is of one-baryon nature, the connected part $\delta H_{1}(z)$ contains two-baryon and possibly three-baryon pieces according to Fig. 4.

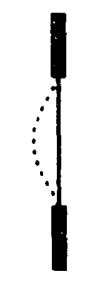

(a)

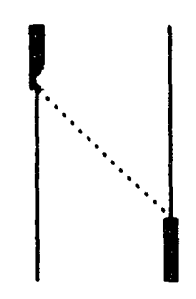

(b)

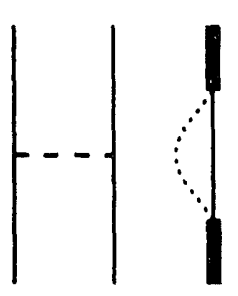

(e)

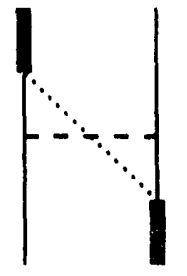

(c)

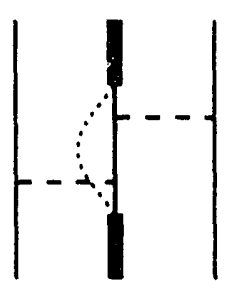

(f)

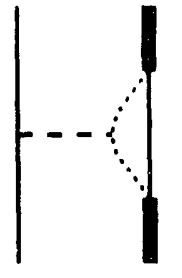

(d)

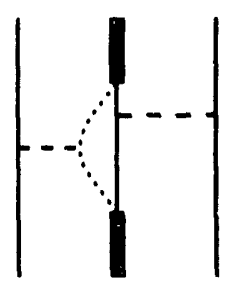

(g)

FIG. 4. Energy-dependent contributions to the effective hamiltonian of Eq. (2.3a) arising írom projecting out the pionic component from the trinucleon wave function. All energy-dependent contributions act in the baryonic Hilbert sector $\mathcal{H}_{\Delta}$ with a $\Delta$-isobar. In the top row the only contribution of one-baryon nature $P \delta H_{0}(z) P$ is shown. Row two (three) gives characteristic examples of two-(three-)baryon nature in $P \delta H_{1}(z) P$. With respect to the pion-nucleon interaction, possible $P_{33}$ background contributions are not considered in this figure. The contributions (c) $-(\mathrm{g})$ disappear, once interactions in the Hilbert sector $\mathcal{H}_{\pi}$ are not taken into account, i.e., $Q H_{1} Q=0$.

Both parts are only defined in the baryonic sectors of Hilbert space and are - in the considered force model - nonzero only in the one with a $\Delta$-isobar, i.e., $P \delta H_{0}(z) P_{N}=$ $P_{N} \delta H_{0}(z) P=P \delta H_{1}(z) P_{N}=P_{N} \delta H_{1}(z) P=0$. They show an energy dependence, though 
the original hamiltonian acts instantaneously without time delay. That energy dependence arises from projecting the pionic component out from the wave function. However, by that energy dependence the pionic component preserves its active presence. Thus, the energy dependence in $\left.\delta H_{0} l z\right)$ and $\delta H_{1}(z)$ is necessary and it is well regulated, always prescribed without any artbitrariness in all applications. This paper only deals with the trinucleon bound state: but clearly the same set of equations (2.3) hold for scattering problems: Due to the energy dependence of the effective baryonic hamiltonian $P\left(H_{0}+\delta H_{0}(z)+H_{1}+\delta H_{1}(z)\right) P$ the wave function components of bound and scattering states. projected onto the baryonic sectors, are not orthogonal. though they belong to states of different three-nucleon energy: However. the formalism naturally restores the orthogonality for the full states which include their pionic components. In the same way only the full bound-state wave function is to be normalized according to Eq. (2.3c). The controlled energy dependence of the effective baryonic hamiltonian is in contrast to a phenomenologically chosen energy dependence of the two-nucleon potential as used once in a while [16]; in the latter case there are no rules which affect the change in the energy dependence to be adopted for different applications.

Despite the energy dependence of the effective hamiltonian $P\left(H_{0}+\delta H_{0}(z)+H_{1}+\right.$ $\left.\delta H_{1}(z)\right) P$ the projected baryonic equation (2.3a) can be decomposed into a set of equations for Faddeev amplitudes in the standard way. We introduce the notation

$$
G_{0}^{P}(z)=\frac{P}{z-P\left[H_{0}+\delta H_{0}(z)\right] P}
$$

for the effective resolvent and

$$
P\left[H_{1}+\delta H_{1}(z)\right] P=\sum_{i} v_{i}(z)+\sum_{i} W_{i}(z)
$$

for the effective baryonic interaction with $v_{i}(z)$ denoting the two-baryon interaction between the pair $(j k)$, (ijk) cyclic, and with $W_{i}(z)$ denoting the three-baryon interaction. particle $i$ being the $\Delta$-isobar in the force before interaction. The effective two-baryon interaction $v_{i}(z)$ has instantaneous contributions arising from $H_{1}$ in and between both baryonic sectors and energy-dependent contributions arising from $\delta H_{1}(z)$ in the Hilbert sector $\mathcal{H}_{\Delta}$ with a $\Delta$-isobar. The effective three-baryon interaction $W_{i}(z)$ of the discussed force model only has energy-dependent contributions arising from $\delta H_{1}(z)$ and consequently it is nonzero only in the baryonic sector $\mathcal{H}_{\Delta}$. Process (e) of Fig. 4 depends on the coordinates of all three baryons, all three of them interact, it therefore yields a true three-baryon force $W_{i}(z)$. However, it is a singular one through the $\delta$-function for the $\Delta$-isobar momentum; in fact, it is unlinked, though it is derived from Eq. (2.4b) and labelled there otherwise. Thus, it has the mathematical structure of a two-body interaction $v_{i}(z)$ in a three-particle Hilbert space. The Appendix A derives the mathematical structure of that particular contribution and shows how it is to be combined with the two-baryon interactions in the calculational treatment.

Using the two-baryon interaction $v_{i}(z)$ for defining the transition matrix

$$
T_{i}(z)=v_{i}(z)\left[1+G_{0}^{P}(z)\right] T_{i}(z)
$$

in the three-baryon space and introducing baryonic Faddeev amplitudes $P\left|\psi_{i}\right\rangle$, i.e., 


$$
P\left|\psi_{i}\right\rangle=G_{0}^{P}\left(E_{B}\right)\left[v_{i}\left(E_{B}\right)+W_{i}\left(E_{B}\right)\right] P\left|\Psi_{B}\right\rangle,
$$

the effective Schrödinger equation (2.3a) gets equivalent to the set of Faddeev equations

$$
\begin{aligned}
P\left|\psi_{i}\right\rangle= & G_{0}^{P}\left(E_{B}\right)\left\{T_{\imath}\left(E_{B}\right)\left[P_{i \jmath k}+P_{i k \jmath}\right]\right. \\
& +\left[1+T_{i}\left(E_{B}\right) G_{0}^{P}\left(E_{E}\right)\right] W_{i}\left(E_{B}\right) \\
& \left.\times\left[1+P_{\imath \jmath k}+P_{i k_{\jmath}}\right]\right\} P\left|\psi_{i}\right\rangle . \\
P\left|\Psi_{B}\right\rangle= & N\left[1+P_{\imath \jmath k}+P_{i k_{\jmath}}\right] P\left|\psi_{i}\right\rangle .
\end{aligned}
$$

The particular form (2.9a) of Faddeev equations is introduced in Ref. [1i] for the case in which explicit and irreducible three-body forces are present. The Faddeev equations (2.9a) greatly simplify, once three-body forces are absent. In Eqs. (2.9) $P_{i j k}$ and $P_{i k \jmath}$ are cyclic and anticyclic permutation operators of three particles $(i j k)$. In Eq. (2.9b) $N$ is a normalization constant which only the normalization condition $(2.3 \mathrm{c})$ for the full wave function determines.

\section{CALCULATIONAL APPARATUS}

Calculations of the trinucleon bound state are carricd out for the force model of Fig. 2 and for variants of it. It has been tested in the two-nucleon system above pion threshold by Refs. [4] and [5]. The force model is used there and for this paper in the approximation $Q H_{1} Q=0$, which neglets all interactions in the pionic sector $\mathcal{H}_{\pi}$ of the Hilbert space.

Since $Q H_{1} Q=0$, all energy-dependent three-baryon contributions to the effective baryonic interaction (2.6) disappear and the Faddeev equations (2.9a) simplify to those with two-baryon forces only, i.e.,

$$
P\left|\psi_{i}\right\rangle=G_{0}^{P}\left(E_{B}\right) T_{i}\left(E_{B}\right)\left[P_{i j k}+P_{i k_{j}}\right] P\left|\psi_{i}\right\rangle .
$$

The only energy-dependent contributions to the effective baryonic hamiltonian (2.3a) which survive are the processes (a) and (b) of Fig. 4. Both are determined by the pion-nucleon$\Delta$ vertex $Q H_{1} P_{\Delta}$ which is calibrated through pion-nucleon scattering in the $P_{33}$ partial wave. The single-baryon nature of the vertex is made explicit by the notation $Q H_{1} P_{\Delta}=$ $\sum_{i} Q h_{1}(i) P_{\Delta}, i$ being the label of the baryon which is transformed from a $\Delta$-isobar to a pion-nucleon state. Process (a) yields the $\Delta$-isobar self-energy correction $P_{\Delta} \delta H_{0}(z) P_{\Delta}$ in the three-baryon resolvent, i.e.,

$$
P_{\Delta} \delta H_{0}(z) P_{\Delta}=\sum_{i} P_{\Delta} h_{1}(i) Q \frac{Q}{\left[z-Q h_{0}(j) Q-Q h_{0}(k) Q\right]-Q h_{0}(i) Q-Q h_{0}(\pi) Q} Q h_{1}(i) P_{\Delta},
$$

process (b) the retarded one-pion exchange $P_{\Delta} \delta H_{1}(z) P_{\Delta}$ in the effective two-baryon interaction, i.e.,

$$
P_{\Delta} \delta H_{1}(z) P_{\Delta}=\sum_{j \neq k} P_{\Delta} h_{1}(k) Q \frac{Q}{\left[z-Q h_{0}(i) Q\right]-Q h_{0}(j) Q-Q h_{0}(k) Q-Q h_{0}(\pi) Q} Q h_{1}(j) P_{\Delta} .
$$


In the single-baryon part $P_{\Delta} \delta H_{0}(z) P_{\Delta}$ the operator $\left[z-Q h_{0}(j) Q-Q h_{0}(k) Q\right]$ of the threebaryon resolvent reflects the fact that at the available energy $z$ the noninteracting nucleons $j$ and $k$ are present and propagate besides the $\lambda-$-isobar $\imath$ : that operator becomes a $c$-number parameter in a three-baryon momentum-space basis. In the two-baryon purt $P_{\Delta} \delta H_{1}(z) P_{\Delta}$ the operator $\left[z-Q h_{0}(i) Q\right]$ of the resolvent reflects the fact that at the available energy $z$ the noninteracting nucleon $i$ is present and propagates beside the interacting nucleon- $\triangle$ pair $(j k)$; that operator becomes a $c$-number parameter in a three-baryon momentum-space basis. Both retarded contributions $P_{\Delta} \delta H_{0}(z) P_{\Delta}$ and $P_{\Delta} \delta H_{1}(z) P_{\Delta}$ are defined in Eqs. (3.2) for the three-baryon system. However. they notice the presence and absence of noninteracting particles through their energy dependence, they are therefore different. e.g., in one-baryon and two-baryon systems. The structure of both parts $P_{\Delta} \delta H_{0}(z) P_{\Delta}$ and $P_{\Delta} \delta H_{1}(z) P_{\Delta}$ and their use in the trinucleon boind-state calculation are now discussed.

\section{A. Three-Baryon Basis States}

The three-baryon basis states $\left|p_{1} q_{1} \nu_{1}\right\rangle$, required for the calculation in the Hilbert sectors $\mathcal{H}_{N}$ and $\mathcal{H}_{\Delta}$ are diagramatically defined in Fig. 5. $\left(p_{1} q_{1}\right)$ are the magnitudes of the Jacobi momenta and $\nu$ abbreviates all discrete quantum numbers.

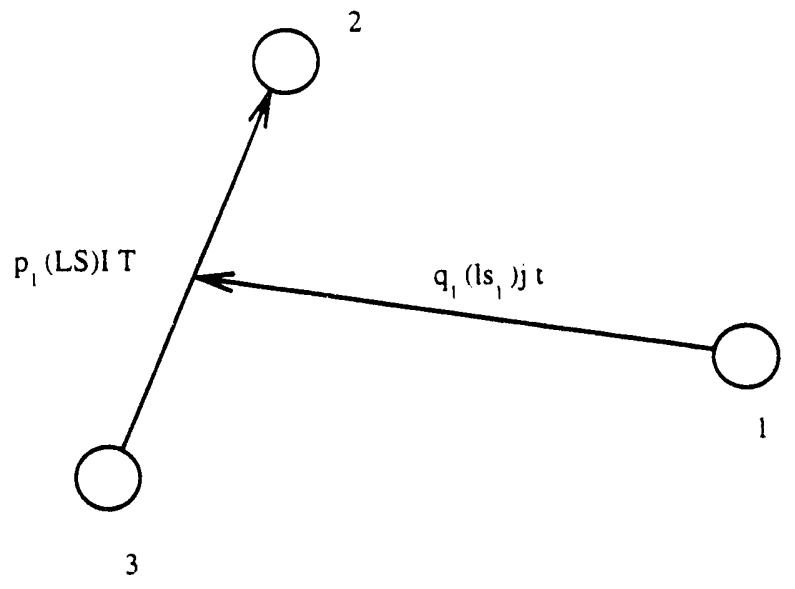

FIG. 5. Three-body Jacobi coordinates. The magnitude of the corresponding momenta are $p_{1}$ and $q_{1}$. In the momentum-space basis states $\left|p_{1} q_{1} \nu_{1}\right\rangle_{1}$ the antisymmetrized state of pair 2 and 3 and the spectator state are coupled with respect to their angular momenta $I$ and $j$ and isospin $T$ and $t_{1}$ i.e., $\left|p_{1} q_{1}\left[(L S) I\left(l s_{1}\right) j\right] \mathcal{J} \mathcal{J}_{z}\left(T t_{1}\right) \mathcal{T} \mathcal{T}_{z}\right\rangle_{1}$. The quantum numbers $J_{i}(l)$ and $S\left(s_{1}\right)$ refer to the orbital angular momentum and spin of the pair (spectator), $\mathcal{J}\left(\mathcal{J}_{z}\right)$ and $\mathcal{T}\left(\mathcal{T}_{z}\right)$ are total angular momentum (projection) and total isospin (projection) of the three-body bound state.

The calculation is done in the trinucleon c.m. system, thus, the total momentum $k$ is zero. In the purely nucleonic Hilbert sector $\mathcal{H}_{N}$ the definition of the Jacobi momenta is standard. In the Hilbert sector $\mathcal{H}_{\Delta}$ the Jacobi momenta are nonrelativistically defined with the mass $m_{N}$ for nucleons and $m_{\Delta}^{0}$ for the $\Delta$-isobar. The momenta $\left(p_{1} q_{1}\right)$ are denoted by $\left(p_{\Delta} q_{\Delta}\right)$ when the $\Delta$-isobar is the spectator particle 1, i.e., 


$$
\begin{aligned}
\mathbf{p}_{\Delta} & =\frac{m_{N} \mathbf{k}_{N 2}-m_{N} \mathbf{k}_{N 3}}{2 m_{N}}, \\
\mathbf{q}_{\Delta} & =\frac{m_{\Delta}^{0}\left(\mathbf{k}_{N_{2}}+\mathbf{k}_{v_{3}}\right)-2 m_{v} \mathbf{k}_{\Delta}}{m_{\Delta}^{0}+2 m_{v}} . \\
\mathbf{k} & =\mathbf{k}_{\Delta}+\mathbf{k}_{N_{2}}+\mathbf{k}_{N_{3}}=0,
\end{aligned}
$$

$$
\begin{aligned}
& H_{\mathrm{C}}\left|p_{\Delta} q_{\Delta} \nu_{\Delta}\right\rangle_{1}= \\
& {\left[2 m_{N}+\frac{p_{\Delta}^{2}}{m_{N}}+\frac{q_{\Delta}^{2}}{4 m_{l_{N}}}+m_{\Delta}^{0}+\frac{q_{\Delta}^{2}}{2 m_{\Delta}^{0}}\right]\left|p_{\Delta} q_{\Delta} \nu_{\Delta}\right\rangle_{1}}
\end{aligned}
$$

with $\mathbf{k}_{\Delta}, \mathbf{k}_{N 2}$ and $\mathbf{k}_{N 3}$ being single-particle n.omenta of the baryons. The momenta $\left(p_{1} q_{1}\right)$ are denoted by $\left(p_{N} q_{N}\right)$ when a nucleon is the spectator particle 1. i.e.,

$$
\begin{aligned}
& \mathbf{p}_{N}=\frac{m_{\Delta}^{0} \mathbf{k}_{N 2}-m_{N} \mathbf{k}_{\Delta}}{m_{N}+m_{\Delta}^{0}} \\
& \mathbf{a}_{N}=\frac{m_{N}\left(\mathbf{k}_{N 2}+\mathbf{k}_{\Delta}\right)-\left(m_{N}+m_{\Delta}^{0}\right) \mathbf{k}_{N 1}}{2 m_{N}+m_{\Delta}^{0}} \\
& \mathbf{k}=\mathbf{k}_{N 1}+\mathbf{k}_{N 2}+\mathbf{k}_{\Delta}=0, \\
& H_{0}\left|p_{N} q_{N} \nu_{N}\right\rangle_{1}= \\
& {\left[m_{N}+m_{\Delta}^{0}+\frac{p_{N}^{3}}{2}\left(\frac{1}{m_{N}}+\frac{1}{m_{\Delta}^{0}}\right)+\frac{q_{N}^{2}}{2\left(m_{N}+m_{\Delta}^{0}\right)}\right.} \\
& \left.\quad+m_{N}+\frac{q_{N}^{2}}{2 m_{N}}\right]\left|p_{N} q_{N} \nu_{N}\right\rangle_{1}
\end{aligned}
$$

The basis states are antisymmetrized with respect to particles 2 and 3 . In the latter case $\left|p_{N} q_{N} \nu_{N}\right\rangle_{1}$ the $\Delta$-isobar is taken to be particle 3 before antisymmetrization. The basis states $\left|p_{\Delta} q_{\Delta} \nu_{\Delta}\right\rangle_{1}$ and $\left|p_{N} q_{N} \nu_{N}\right\rangle_{1}$ are orthogonal to each other; they are different states in the complete set of states describing two nucleons and one $\Delta$-isobar.

\section{B. The $\Delta$-Isobar Self-Energy Correction $P_{\Delta} \delta H_{0}(z) P_{\Delta}$ and the Effective Three-Baryon Resolvent}

The effective three-baryon resolvent (2.5) is illustrated in Fig. 6. 


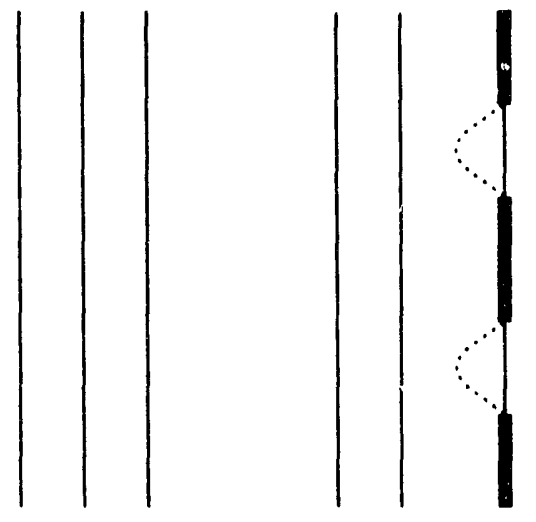

FIG. 6. Effective three-baryon resolvent (2.5). Its form ir the Hilbert sector $\mathcal{H}_{N}$ is diagramatically shown on the left side and its form in the Hilbert sector $\mathcal{H}_{\Delta}$ on the right side.

It is trivial in the Hilbert sector $\mathcal{H}_{N}$, but receives the pionic correction $P_{\Delta} \delta H_{0}(z) P_{\Delta}$ in the sector $\mathcal{H}_{\Delta}$ with one $\Delta$-isobar. That pionic correction is also seen in $P_{33}$ pion-nucleon scattering as :llustrated in Fig. 7, and it is calibrated there.

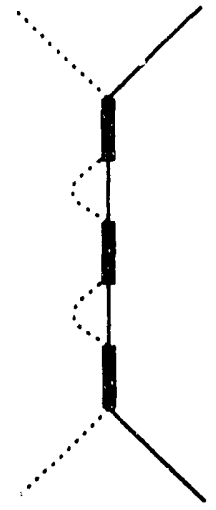

FIG. 7. Characteristic contribution to the pion-nucleon transition matrix in the $P_{33}$ partial wave. The force model of Fig. 2 does not have any additional background potential $Q H_{1} Q$ in that partial wave. An effective propagation of the $\Delta$-isobar can be read off from the transition matrix and reoccurs - together with the propagation of two additional nucleons - in the effective three-baryon resolvent (2.5) of Fig. 6 .

The pion-nucleon transition matrix in the $P_{33}$ partial wave is

$$
\begin{aligned}
t\left(z_{\Delta}, k_{\Delta}\right) & =Q h_{1}(i) P_{\Delta} \\
& \times \frac{P_{\Delta}}{z_{\Delta}-m_{\Delta}^{0}-\frac{k_{\Delta}^{2}}{2 m_{\Delta}^{0}}-P_{\Delta} h_{1}(i) Q \frac{Q}{z_{\Delta}-Q h_{0}(i) Q-Q h_{0}(\pi) Q} Q h_{1}(i) P_{\Delta}} P_{\Delta} h_{1}(i) Q,
\end{aligned}
$$




$$
t\left(z_{\Delta}, k_{\Delta}\right)=|f\rangle \frac{1}{z_{\Delta}-M_{\Delta}\left(z_{\Delta} \cdot k_{\Delta}\right)-\frac{k^{2}}{2 m_{\Delta}}+\frac{1}{2} \Gamma_{\Delta}\left(z_{\Delta} \cdot k_{\Delta}\right)}\langle f|
$$

with $Q h_{1}(i) P_{\Delta}=|f\rangle . P_{\Delta} h_{1}(i) Q=\langle f|$. Pion-nucleon relative and c.m. momenta. i.e.. $\pi$ and $k_{د}$. are introduced by

$$
\begin{aligned}
\boldsymbol{\pi} & =\frac{\omega_{-}\left(k_{\pi}\right) \mathbf{k}_{N}-m_{N} \mathbf{k}_{\pi}}{m_{N}+\nu_{\pi}\left(k_{\pi}\right)} . \\
\mathbf{k}_{\Delta} & =\mathbf{k}_{N}+\mathbf{k}_{\pi} .
\end{aligned}
$$

Since the pion is treated relativistically and the nucleon nonrelativistically, the reduction of operators from a many-baryon to a single-baryon form and, conversely. the embedding of a sing!e-baryon operator in many-baryon systems can often be done only approximately. We use the approximation

$$
\begin{aligned}
& Q h_{0}(i) Q+Q h_{0}(\pi) Q \\
& =m_{N}+\frac{k_{. v}^{2}}{2 m_{. N}}+\sqrt{m_{\pi}^{2}+k_{\pi}^{2}} \\
& \approx m_{N}+\frac{\pi^{2}}{2 m_{N}}+\sqrt{m_{\pi}^{2}+\pi^{2}}+\frac{k_{\Delta}^{2}}{2\left(m_{N}+\sqrt{m_{\pi}^{2}+\pi^{2}}\right)} \\
& =Q h_{\text {orel }}^{\pi . v}(i) Q+\frac{k_{\Delta}^{2}}{2\left(m_{N}+\sqrt{m_{\pi}^{2}+\pi^{2}}\right)},
\end{aligned}
$$

which avoids angles between the three-momenta $\boldsymbol{\pi}$ and $\mathbf{k}_{\Delta}$ in the kinetic energy operator: it is employea in the step from Eq. (3.7a) to Eq. (3. Tb). and it is believed to be quite accurate. The kinetic energy operator of relative pion-nucleon motion $Q h_{0 \text { rel }}^{\pi N}(i) Q$ is introduced. Thus. the effective mass $h_{\Delta}\left(z_{\Delta}, k_{\Delta}\right)$ and the effective width $\Gamma_{\Delta}\left(z_{\Delta}, k_{\Delta}\right)$ of the $\Delta$-isobar with momentum $\mathbf{k}_{\Delta}$ yield that pionic correction $P_{\Delta} \delta H_{0}(z) P_{\Delta}$. once the propagation of two additional nucleons in $P_{\Delta} \delta H_{0}(z) P_{\Delta}$ is taken into account according to Eq. (3.2a). In a three-baryon system with single $\Delta$-isobar excitation

$$
\begin{aligned}
& P_{\Delta}\left[H_{0}+\delta H_{0}(z)\right] P_{\Delta}\left|p_{\Delta} q_{\Delta} \nu_{\Delta}\right\rangle_{1}=\left[2 m_{N}+\frac{p_{\Delta}^{2}}{m_{N}}+\frac{q_{\Delta}^{2}}{4 m_{N}}+M_{\Delta}\left(z-2 m_{N}-\frac{p_{A}^{2}}{m_{N}}-\frac{q_{\Delta}^{2}}{4 m_{N}}, q_{\Delta}\right)\right. \\
& \left.+\frac{q_{\Delta}^{2}}{2 m_{\Delta}^{0}}-\frac{i}{2} \Gamma\left(z-2 m_{N}-\frac{p_{\Delta}^{2}}{m_{N}}-\frac{q_{\Delta}^{2}}{4 m_{N}}, q_{\Delta}\right)\right]\left|p_{\Delta} q_{\Delta} \nu_{\Delta}\right\rangle_{1} .
\end{aligned}
$$

This paper carries out a trinucleon bound-state calculation only, thus, the required threebaryon available energy $z$ is always smaller than $3 m_{N}$. As a consequence, the single-baryon available energy in the effective mass and width of the $\Delta$-isobar according to Eq. (3.9a) is with $z-2 m_{N}-p_{\Delta}^{2} / m_{N}-q_{\Delta}^{2} / 4 m_{N}<m_{N}<m_{N}+m_{\pi}$. The width $\Gamma_{\Delta}\left(z_{\Delta}, k_{\Delta}\right)$ therefore does not contribute to the effective three-baryon resolvent (2.5), which in the Hilbert sector $\mathcal{H}_{\Delta}$ takes the form

$$
\begin{aligned}
& { }_{1}\left\langle p_{\Delta}^{\prime} q_{\Delta}^{\prime} \nu_{\Delta}^{\prime}\left|G_{0}^{P}(z)\right| p_{\Delta} q_{\Delta} \nu_{\Delta}\right\rangle_{1}=\frac{\delta\left(p_{\Delta}^{\prime}-p_{\Delta}\right)}{p_{\Delta}^{2}} \frac{\delta\left(q_{\Delta}^{\prime}-q_{\Delta}\right)}{q_{\Delta}^{2}} \delta_{\nu_{\Delta}^{\prime} \nu_{\Delta}} \\
& \times\left[z-2 m_{N}-\frac{p_{\Delta}^{2}}{m_{N}}-\frac{z_{\Delta}^{2}}{2}\left(\frac{1}{2 m_{N}}+\frac{1}{m_{\Delta}^{0}}\right)-H_{\Delta}\left(z-2 m_{N}-\frac{p_{\Delta}^{2}}{m_{N}}-\frac{q_{\Delta}^{2}}{4 m_{N}}, q_{\Delta}\right)\right]^{-1}
\end{aligned}
$$


for the considered available energies $z$ and for the basis states $\left|p_{\Delta} q_{\Delta} \nu_{\Delta}\right\rangle_{1}$ of Eq. (3.4).

The trinucleon bound-state calculation requires the operators in the Hilbert sector $\mathcal{H}_{\Delta}$ also in the basis $\left|p_{. v} q_{N}^{\prime} \nu_{N}\right\rangle_{1}$ of Eq. (3.6). Compared with Eq. (3.9b) the three-baryon resolvent is more complicated, since nondiagonal, in this basis. In order to simplify calculations, we opted for an approximation which also makes ${ }_{1}\left\langle p_{N}^{\prime} q_{N}^{\prime} \nu_{N}^{\prime}\left|G_{0}^{P}(z)\right| p_{N} q_{N} \nu\right\rangle_{1}$ diagonal in the momenta and channels of the basis states $\left|p_{N} q_{N} \nu_{N}\right\rangle_{1}$. The approximation is best seen in the quantity

$$
\begin{aligned}
& \frac{1}{z-Q H_{0} Q} Q H_{1} P_{\Delta} \mid p_{N} q_{\left.N \nu_{N}\right\rangle_{1}} \\
= & \frac{Q}{\left[z-Q h_{0}(1) Q-Q h_{0}(2) Q\right]-Q h_{0}(3) Q-Q h_{0}(\pi) Q} Q h_{1}(3) P_{\Delta}\left|p_{N} q_{N} \nu_{N}\right\rangle_{1} \\
= & \frac{1}{z-2 m_{N}-\frac{k_{N 1}^{2}}{2 m_{N}}-\frac{k_{N 2}^{2}}{2 m_{N}}-\frac{k_{\Delta}^{2}}{2 m_{\Delta}}-\left[Q h_{0}(3) Q-Q h_{0}(\pi) Q-\frac{k_{\Delta}^{2}}{2 m_{\Delta}^{\sigma}}\right]} Q h_{1}(3) P_{\Delta}\left|p_{N} q_{N} \nu_{N}\right\rangle_{1} \\
\approx & \frac{1}{z-2 m_{N}-\frac{k_{N 1}^{2}}{2 m_{N}}-\frac{k_{N 2}^{2}}{2 m_{N}}-\frac{k_{2}^{2}}{2 m_{\Delta}^{0}}-Q h_{0 \mathrm{rel}}^{\pi N}(3) Q} Q h_{1}(3) P_{\Delta}\left|p_{N} q_{N} \nu_{N}\right\rangle_{1}
\end{aligned}
$$

The $\Delta$-isobar carries the baryon label 3 . The baryon kinetic energy operator $k_{N_{1}}^{2} / 2 m_{N}+$ $k_{.22}^{2} / 2 m_{N}+k_{\Delta}^{2} / 2 m_{\Delta}^{0}$ without rest masses is rewritten in terms of the Jacobi momenta $\left(\mathbf{p}_{N} \mathbf{q}_{N}\right)$ of Eq. (3.5), whereas $\left[Q h_{0}(3) Q-Q h_{0}(\pi) Q-k_{\Delta}^{2} / 2 m_{\Delta}^{0}\right]$ is approximated in the last step of Eq. (3.10) by the pion-nucleon relative kinetic energy $Q h_{0}^{\pi N}(3) Q$ including rest masses. According to Eq. (3.8c) $Q h_{0}(3) Q+Q h_{0}(\pi) Q$ has a c.m. contribution $k_{\Delta}^{2} / 2\left(m_{N}+\sqrt{m_{\pi}^{2}+\pi^{2}}\right)$ with $k_{\Delta}^{2}$ not being a function of the magnitudes $p_{N}$ and $q_{N}$ of the Jacobi momenta only, but also of the angle between them; the c.m. dependence couples partial waves $\nu_{N}$ in a nontrivial way. Once that c.m. contribution is accounted for by $k_{\Delta}^{2} / 2 m_{\Delta}^{0}$ with sufficient accuracy, the basis states $\left|p_{N} q_{N} \nu_{N}\right\rangle_{1}$ become true eigenstates of the single-baryon part $H_{0}+\delta H_{0}(z)$ in the effective hamiltonian, i.e.,

$$
\begin{aligned}
& P_{\Delta}\left[H_{0}+\delta H_{0}(z)\right] P_{\Delta}\left|p_{N} q_{N} \nu_{N}\right\rangle_{1}=\left[2 m_{N}+\frac{p_{N}^{2}}{2}\left(\frac{1}{m_{N}}+\frac{1}{m_{\Delta}^{0}}\right)+\frac{q_{N}^{2}}{2}\left(\frac{1}{m_{N}+m_{\Delta}^{0}}+\frac{1}{m_{N}}\right)\right. \\
& +M_{\Delta}\left(z-2 m_{N}-\frac{p_{N}^{2}}{2}\left(\frac{1}{m_{N}}+\frac{1}{m_{\Delta}^{0}}\right)-\frac{q_{N}^{2}}{2}\left(\frac{1}{m_{N}+m_{\Delta}^{0}}+\frac{1}{m_{N}}\right), 0\right) \\
& \left.-\frac{i}{2} \Gamma_{\Delta}\left(z-2 m_{N}-\frac{p_{N}^{2}}{2}\left(\frac{1}{m_{N}}+\frac{1}{m_{\Delta}^{0}}\right)-\frac{q_{N}^{2}}{2}\left(\frac{1}{m_{N}+m_{\Delta}^{0}}+\frac{1}{m_{N}}\right), 0\right)\right]\left|p_{N} q_{N} \nu_{N}\right\rangle_{1} .
\end{aligned}
$$

The approximation (3.10) employs the effective mass $M_{\Delta}\left(z_{\Delta}, k_{\Delta}\right)$ and the effective width $\Gamma_{\Delta}\left(z_{\Delta}, k_{\Delta}\right)$ of the $\Delta$-isobar at the momentum $k_{\Delta}=0$, though the pion-nucleon system is not at rest in a three-baryon system; the reason is that the approximation (3.10) works with the pion-nucleon relative kinetic energy operator $Q h_{0 \text { rel }}^{\pi N} Q$ and pushes the dependence on the moving pion-nucleon c.m. into an appropriate available energy $z_{\Delta}$. The approximation (3.10) also assumes baryon 3 to be the $\Delta$-isobar; however, the result (3.11a) is symmetric in baryons 2 and 3 and therefore applies to the basis state $\left|p_{N} q_{N} \nu_{N}\right\rangle_{1}$ antisymmetrized with 
respect to baryons 2 and 3 . Using the result (3.11a) the effective three-baryon resolvent (2.j) becomes diagonal also in the basis states $\left|p_{N} q_{N} \nu_{N}\right\rangle_{1}$ of the Hilbert sector $\mathcal{H}_{\Delta}$ and takes the form

$$
\begin{aligned}
& { }_{1}\left\langle p_{N}^{\prime} q_{. N}^{\prime} \nu_{N}^{\prime}\left|G_{0}^{P}(z)\right| p_{N} q_{N} \nu_{N}\right\rangle_{1}=\frac{\delta\left(p_{. N}^{\prime}-p_{N}\right)}{p_{N}^{2}} \frac{\delta\left(q_{N}^{\prime}-q_{N}\right)}{q_{N}^{2}} \delta_{\nu_{N}^{\prime} \nu_{N}} \\
\times & {\left[z-2 m_{. N}-\frac{p_{N}^{2}}{2}\left(\frac{1}{m_{. N}}+\frac{1}{m_{\Delta}^{0}}\right)-\frac{q_{N}^{2}}{2}\left(\frac{1}{m_{. N}+m_{\Delta}^{0}}+\frac{1}{n_{. N}}\right)\right.} \\
- & \left.M_{\Delta}\left(z-2 m_{N}-\frac{p_{N}^{2}}{2}\left(\frac{1}{m_{N}}+\frac{1}{m_{\Delta}^{0}}\right)-\frac{q_{N}^{2}}{2}\left(\frac{1}{m_{N}+m_{\Delta}^{0}}+\frac{1}{m_{N}}\right), 0\right)\right]^{-1}
\end{aligned}
$$

for the available energy $z$ considered in the trinucleon bound state.

Due to its energy dependence, the $\Delta$-isobar self-energy correction $P_{\Delta} \delta H_{0}(z) P_{\Delta}$ is different in a two-baryon system: It notices the absence of the third baryon. When described by the basis states $\left|p_{N} \nu_{N}\right\rangle$ for particles 2 and 3 in the basis of Eq. (3.6), it becomes

$$
\begin{aligned}
P_{\Delta}\left[H_{0}+\delta H_{0}^{[2]}\left(z_{N \Delta}\right)\right]\left|p_{N} \nu_{N}\right\rangle & =\left[m_{N}+\frac{p_{N}^{2}}{2}\left(\frac{1}{m_{N}}+\frac{1}{m_{\Delta}^{0}}\right)\right. \\
& +M_{\Delta}\left(z_{N \Delta}-m_{N}-\frac{p_{N}^{2}}{2}\left(\frac{1}{m_{N}}+\frac{1}{m_{\Delta}^{0}}\right), 0\right) \\
& \left.-\frac{i}{2} \Gamma_{\Delta}\left(z_{N \Delta}-m_{N}-\frac{p_{N}^{2}}{2}\left(\frac{1}{m_{N}}+\frac{1}{m_{\Delta}^{0}}\right), 0\right)\right]\left|p_{N} \nu_{N}\right\rangle
\end{aligned}
$$

The approximation of Eq. (3.10) on the pion-nucleon c.m. kinetic energy is used accordingly. The superscript [2] indicates that the self-energy correction $P_{\Delta} \delta H_{0}^{[2]}\left(z_{N \Delta}\right) P_{\Delta}$ refers to a twobaryon c.m. system: it will be needed for the actual realization of the employed force model in Subsect. D.

The validity of the approximation (3.10), important for the three-baryon resolvent (3.11b) and for the self-energy correction (3.12) in the two-baryon system, is proven as follows: The approximating step in Eq. (3.10) is not carried out, $\left[Q h_{0}(3) Q+Q h_{0}(\pi) Q-\mathbf{k}_{\Delta}^{2} /\left(2 m_{\Delta}^{0}\right)\right]$ is used full, the dependence of $\mathbf{k}_{\Delta}$ on the angle between $\mathbf{p}_{N}$ and $\mathbf{q}_{N}$ is kept; however, that angle is assumed to be fixed at values 0 or $\pi / 2$ or $\pi$, respectively, thus, $\mathbf{k}_{\Delta}$ again remains effectively dependent on the magnitudes of the Jacobi momenta $p_{N}$ and $q_{N}$ only and does not yield any channel coupling. Using approximation (3.10) and the three different approximations indicated in this paragraph in trinucleon calculations, the trinucleon binding energy varies by less than $1 \mathrm{keV}$, i.e., within the numerical accuracy; the approximation (3.10) is henceforth considered quite satisfactory.

\section{Retarded One-Pion Exchange $P_{\Delta} \delta H_{1}(z) P_{\Delta}$}

The retarded one-pion exchange $P_{\Delta} \delta H_{1}(z) P_{\Delta}$ of the effective baryonic hamiltonian in Eq. (2.3a) is illustrated by process (b) of Fig. 4. It is defined in Eq. (3.2b) for the threebaryon system. Refs $[4,5]$ test the employed force model in the two-nucleon system above pion threshold. Thus, the retarded one-pion exchange $P_{\Delta} \delta H_{1}(z) P_{\Delta}$ is also needed in the 
two-baryon c.m. system. It is different there. since it notices the absence of the third noninteracting nucleon. It is notationally differentiated as $P_{\Delta} \delta H_{1}^{[2]}\left(z_{N \Delta}\right) P_{\Delta}$ by the superscript [2]. It takes the form

$$
\begin{aligned}
P_{\Delta} \delta H_{1}^{[2]}\left(z_{N \Delta}\right) P_{\Delta} & =v^{v \Delta \rightarrow \Delta N}\left(z_{N \Delta}\right) \\
v^{N \Delta-\Delta N}\left(z_{N \Delta}\right) & =\sum_{\substack{j, k=2.3 \\
j \neq k}} P_{\Delta} h_{1}(k) Q \\
& \times \frac{Q}{z_{N \Delta}-\left[Q h_{0}(2) Q+Q h_{0}(3) Q+Q h_{0}(\pi) Q-\frac{\left(\mathbf{k}_{N 2}+\mathbf{k}_{N 3}+\mathbf{k}_{\pi}\right)^{2}}{2 m_{N}+\sqrt{m_{\pi}^{2}+k_{\pi}^{2}}}\right]} Q h_{1}(j) P_{\Delta}
\end{aligned}
$$

It can be calculated in the basis $\left|p_{N} \nu_{N}\right\rangle$ used in Eq. (3.12) for the corresponding $\Delta$-isobar self-energy correction $P_{\Delta} \delta H_{0}^{[2]}\left(z_{N \Delta}\right) P_{\Delta}$ in the two-baryon system; the explicit form of its matrix elements is given in Ref. [14]. When embedding the retarded one-pion exchange into a three-baryon system, the approximation $m_{N}+\sqrt{m_{\pi}+k_{\pi}^{2}} \approx m_{\Delta}^{0}$ is used as in Eq. (3.10) for the total mass of the interacting pion-nucleon system. Its relation to the same process in the two-nucleon system can then be given, i.e..

$$
\begin{aligned}
& { }_{1}\left\langle p_{N}^{\prime} q_{N}^{\prime} \nu_{N}^{\prime}\left|P_{\Delta} \delta H_{1}(z) P_{\Delta}\right| p_{N} q_{N} \nu_{N}\right\rangle_{1}=\frac{\delta\left(q_{N}^{\prime}-q_{N}\right)}{q_{N}^{2}} \\
\times & \left\langle p_{N}^{\prime} \nu_{N}^{\prime}\left|v^{N \Delta \rightarrow \Delta N}\left(z-m_{N}-\frac{q_{N}^{2}}{2}\left(\frac{1}{m_{N}}+\frac{1}{m_{N}+m_{\Delta}}\right)\right)\right| p_{N} \nu_{N}\right\rangle .
\end{aligned}
$$

The three-baryon basis $\left|p_{N} q_{N} \nu_{N}\right\rangle_{1}$ of Eq. (3.5) is the appropriate one for a nucleon- $د$ interaction in a three-baryon system. We note that the energy dependence in the retarded one-pion exchange $P_{\Delta} \delta H_{1}(z) P_{\Delta}$ has a precise meaning and changes the retarded interaction in a controlled way depending on the many-baryon system into which it is embedded.

\section{Parametrization of the Interaction Hamiltonian (2.1)}

The interaction hamiltonian is the force model of Fig. 2 with active pion and $\Delta$-isobar degrees of freedom. Those degrees of freedom only become active in isospin-triplet partial waves; in isospin-singlet partial waves the interaction is purely nucleonic and represented solely by process (a) of Fig. 2. In this paper it is assumed that the interaction hamiltonian $H_{1}$ vanishes in the pionic sector, i.e., $Q H_{1} Q=0$. In general, that is a physically severe assumption, employed already in Ref. [4]: E.g., in the presence of a pion two nucleons cannot be bound; thus, all pion-deuteron processes are not described internally consistent under such an assumption.

Two distinct parametrizations, labelled by $H(1)$ and $S(1)$ in the tables and the result section, are chosen for the baryonic interaction, which differ by their forms of the pionand rho-exchange transition potential $P_{\Delta} H_{1} P_{N}$ : The parametrization with the transition potential of Ref. [10] used there for the force model is labelled $H$ here, since it is based on rather hard form factors, whereas that with the transition potential of Refs. [4,5] based on rather soft form factors is labelled by $S$. 
The instantaneous nucleon- $\Delta$ potential $P_{\Delta} H_{1} P_{\Delta}$ is that of Refs. [4.18]; its exchange part illustrated in Fig. 2(c) is based on pion and rho exchange; only half of the full pion exchange is kept in $P_{\Delta} H_{1} P_{\Delta}$, since the other half, denoted by $\frac{1}{2} \pi_{R}$ in Table I. is generated explicitly by the force model as $P_{\Delta} \delta H_{1}(z) P_{\Delta}$ in a retarded fashion according to Eq. (3.14); the subscript $R$ in the notation $\frac{1}{2} \pi_{R}$ indicates its retardation. The unretarded half of the pion exchange, kept in $P_{\Delta} H_{1} P_{\Delta}$. is identified with the on-shell form $v^{N \Delta-\Delta N}\left(z_{N \Delta_{0}}\right)$ of Eq. (3.13a) as in Refs. [14,4]; it is denoted by $\frac{1}{2} \pi_{S}$ in Table I, the subscript $S$ in the notation $\frac{1}{2} \pi_{S}$ indicates that it is unretarded, but based on the soft form factors of the retarded pion-exchange $P_{\Delta} \delta H_{1}(z) P_{\Delta}$.

In contrast to Refs. [4.5] the nucleonic part of the interaction is chosen as

$$
P_{N} H_{1} P_{N}=V_{N N}-P_{. N} H_{1} P_{\Delta} \frac{P_{\Delta}}{2 m_{N}-P_{\Delta}\left[H_{0}+\delta H_{0}^{[2]}\left(2 m_{N}\right)+H_{1}+\delta H_{1}^{[2]}\left(2 m_{N}\right)\right] P_{\Delta}} P_{\Delta} H_{1} P_{. v}
$$

The choice (3.15) yields exact phase equivalence at zero kinetic energy and approximate phase equivalence at low kinetic energies between the full force model and a realistic, but purely nucleonic reference potential $V_{N N}$. The Paris Potential [19] is chosen as reference potential $V_{N N}$. That reference potential is employed in all isospin-singlet partial waves. The choice (3.15) is a conceptual improvement compared to Ref. [4]. The improved phase equivalence is documented in Ref. [20] which also demonstrates that that improvement is quantitatively irrelevant for observables of the two-nucleon system above pion threshold.

\section{E. Solution of Trinucleon Equations}

The Faddeev equations (3.1) are solved in momentum space using the technical apparatus of Refs. [10] and [13]. The two-baryon interaction is assumed to act in all partial waves up to total pair angular momentum $I=2.18$ purely nucleonic Faddeev amplitudes $\left.P_{. v} \mid \psi_{i}\right)$ arise in the partial-wave decomposition defined by the Jacobi coordinates and discrete quantum numbers of Fig. 5 ; in addition, 14 Faddeev amplitudes $P_{\Delta}\left|\psi_{i}\right\rangle$ with a single $\Delta$-isobar in the pair and one with the $\Delta$-isobar as spectator are taken into account as in Refs. $[10,13]$. The employed discretization of the equations (3.1) is the one of Ref. [13]. The triton binding energy $E_{T}$, and the baryonic components $P\left|\Psi_{B}\right\rangle$ of the wave function according to Eq. (2.9b), are obtained from such a calculation for the force model defined in Subsect. D. The pionic component $Q\left|\Psi_{B}\right\rangle$ of the wave function can in principle be gotten from the baryonic ones $P\left|\Psi_{B}\right\rangle$ by Eq. (2.3b); however, this paper only computes its weight $\left\langle\Psi_{B}|Q| \Psi_{B}\right\rangle$ in the trinucleon wave function according to

$$
\begin{aligned}
& \left\langle\Psi_{B}|Q| \Psi_{B}\right\rangle=\left\langle\Psi_{B}\left|P_{\Delta} H_{1} Q \frac{Q}{\left(E_{B}-Q H_{0} Q\right)^{2}} Q H_{1} P_{\Delta}\right| \Psi_{B}\right\rangle \\
& =\left.\left\langle\Psi_{B}\left|P_{\Delta}\left(-\frac{\partial}{\partial z}\right)\left[\delta H_{0}(z)+\delta H_{1}(z)\right] P_{\Delta}\right| \Psi_{B}\right\rangle\right|_{z=E_{B}} \\
& \left\langle\Psi_{B}|Q| \Psi_{B}\right\rangle=3 \sum_{\nu} \int p_{\Delta}^{2} d p_{\Delta} q_{\Delta}^{2} d q_{\Delta}\left\langle\Psi_{B} \mid p_{\Delta} q_{\Delta} \nu_{\Delta}\right\rangle_{1}
\end{aligned}
$$




$$
\begin{aligned}
& \times\left.\left(-\frac{\partial}{\partial z_{\Delta}}\right) M_{\Delta}\left(z_{\Delta}, q_{\Delta}\right)_{1}\left\langle p_{\Delta} q_{\Delta} \nu_{\Delta} \mid \Psi_{B}\right\rangle\right|_{z_{\Delta}=E_{T}+m_{. N}-\frac{p_{\Delta}^{2}}{m_{. N}}-\frac{q_{\Delta}^{2}}{4 m_{N}}} \\
& +3 \sum_{\nu^{\prime} \nu} \int p_{. V}^{\prime 2} d p_{N}^{\prime} p_{N}^{2} d p_{.} q_{. V}^{2} d q_{N}\left\langle\Psi_{B} \mid p_{N}^{\prime} q_{.}^{\prime} \nu_{. V}^{\prime}\right\rangle_{1}
\end{aligned}
$$

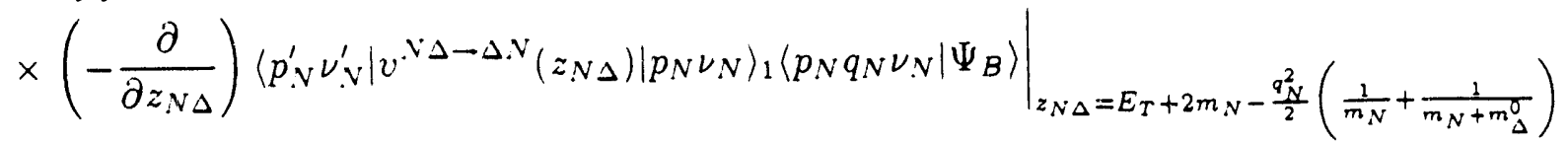

\section{F. Comparison with Coupled-Channel Calculations}

The force model of this paper, defined in Fig. 2 and employed in the calculation of the trinucleon bound state, is used in two parametrizations as Subsect. D describes. In the force model, the bare $\Delta$-isobar is dynamically coupled to pion-nucleon states and builds up, by that coupling, the physical $P_{33}$ resonance in the nuclear medium. We say the force model is based on a dynamic $\Delta$-isobar. The calculation of that full force model is compared with coup'ed-channel calculations of the trinucleon bound state in which the $\Delta$-isobar does not couple to pion-nucleon states, keeps a fixed mass $m_{\Delta}^{0}$ without any pionic correction, i.e., $P_{\Delta} \delta H_{0}(z) P_{\Delta}=0$, and interacts with a nucleon only through unretarded potentials, i.e., $P_{\Delta} \delta H_{1}(z) P_{\Delta}=0$. In those coupled-channel calculations $Q H_{1} P_{\Delta}=P_{\Delta} H_{1} Q=0$, thus. the trinucleon bound state does not have any pionıc components either, i.e., $Q\left|\Psi_{B}\right\rangle=0$. We say those coupled-channel rariants of the full force model are based on a stable $\Delta$-isobar. The technical apparatus which this paper borrows from Refs. [10,13] was originally designed for those coupled-channel calculations.

The aim of this paper is to find the validity of coupled-channel approximations to the full force model. This is the reason why various choices for the stable $\Delta$-isobar mass $m_{\Delta}^{0}$ and for the instantaneous pion exchange between the $\Delta$-isobar and a nucleon are tried out. The various choices are listed in Table I and are described - together with the trinucleon results - in Sect. IV. 
TABLE I. Employed Force Models with $\Delta$-Isobar and Pion Degrees of Freedom

\begin{tabular}{|c|c|c|c|c|c|}
\hline & Bare $\Delta$-Mass & Effective $\Delta$-Mass & $P_{\Delta} H_{1} P_{N}$ & \multicolumn{2}{|c|}{$\begin{array}{c}P_{\Delta}\left[H_{1}+\delta H_{1} !=\|\right] P_{\Delta} \\
\pi-\text { Exchange. Fig. } 2(\mathrm{c})\end{array}$} \\
\hline$\overline{H(1)}$ & 1315 & $M_{\Delta}\left(z_{\Delta} \cdot k_{\Delta}\right)$ & $H[10]$ & $\frac{1}{2} \pi_{R}+\frac{1}{2} \pi_{S}$ & [4] \\
\hline$H(2)$ & 1290 & $m_{\Delta}^{0}$ & $H[10]$ & $\pi_{S}$ & [4] \\
\hline$H(3)$ & 1232 & $m_{\Delta}^{0}$ & $H[10]$ & $\pi_{S}$ & [4] \\
\hline$H(4)$ & 1232 & $m_{\Delta}^{0}$ & $H[10]$ & $\pi_{H}$ & [18] \\
\hline$\overline{S(1)}$ & 1315 & $M_{\Delta}\left(z_{\Delta}, k_{\Delta}\right)$ & $S[4]$ & $\frac{1}{2} \pi_{R}+\frac{1}{2} \pi_{S}$ & {$[t]$} \\
\hline$S(2)$ & 1290 & $m_{\Delta}^{0}$ & $S[4]$ & $\pi_{S}$ & {$[-4]$} \\
\hline$S(3)$ & 1232 & $m_{\Delta}^{0}$ & $S[4]$ & $\pi_{S}$ & {$[4]$} \\
\hline$S(4)$ & 1232 & $m_{\Delta}^{\overrightarrow{0}}$ & $S[4]$ & $\pi_{H}$ & {$[18]$} \\
\hline
\end{tabular}




\section{RESULTS}

Calculations of the form which Refs. [4,5] and this paper report on have three physics objectives in mind:

1. The full force model of Fig. 2 should be tuned in the two-nucleon system above pion threshold. In particular, that tuning process should fix the strength and shape of the two-baryon transition potential $P_{\Delta} H_{1} P_{N}$ from two-nucleon to nucleon- $\Delta$ states and of the nucleon- $\Delta$ potential $P_{\Delta} H_{1} P_{\Delta}$, potentials on which one lacks detailed information otherwise.

2. The calculation of the trinucleon bound state should determine the amount of threenucleon force arising from the explicit excitation of a $\Delta$-isobar and from the explicit production of a pion in the trinucleon bound state.

3. The conditions under which the simpler coupled-channel calculations for the trinucleon bound state approximate the results of the full force model with a dynamic $\Delta$-resonance are to be found.

This paper follows that ambitious program, though it is unable to carry it through to full satisfaction. It uses two versions of the force model of Fig. 2 with the approximation $Q H_{1} Q=0$. That approximation is an inconsequential one for the trinucleon bound state, as the results will prove, but a fatal one, if serious tuning of the force model to all observables in the two-nucleon system above pion threshold were attempted.

TABLE II. Results for some trinucleon bound state properties. The computed binding energies are correct within $10 \mathrm{keV}$ only. Thus, the last digit in rows $E_{T}, \Delta E_{2}$ and $\Delta E_{3}$ of this table are not significant on an absolute scale. The last digit is, however, significant for relative changes, and this is the reason why it is quoted - against our practice in other papers. The nucleonic probabilities $P_{\mathcal{L}}$ in the wave function are split up according to total orbital angular momentum $\mathcal{L}$ and for $\mathcal{L}=0$ also according to the symmetry properties of the orbital wave function components in the standard way.

\begin{tabular}{lrrrrrrrrr}
\hline \hline & Paris & $H(1)$ & $H(2)$ & $H(3)$ & $H(4)$ & $S(1)$ & $S(2)$ & $S(3)$ & $S(4)$ \\
\hline$E_{T}[\mathrm{MeV}]$ & -7.381 & -7.849 & -7.866 & -7.885 & -7.912 & -7.627 & -7.636 & -7.643 & -7.667 \\
$\Delta E_{2}[\mathrm{MeV}]$ & - & 0.456 & 0.425 & 0.494 & 0.460 & 0.248 & 0.227 & 0.272 & 0.258 \\
$\Delta E_{3}[\mathrm{MeV}]$ & - & -0.924 & -0.910 & -0.998 & -0.991 & -0.494 & -0.482 & -0.534 & -0.544 \\
$P_{S}[\%]$ & 90.13 & 88.23 & 88.35 & 88.06 & 88.20 & 89.05 & 89.13 & 88.95 & 89.00 \\
$P_{S^{\prime}}[\%]$ & 1.40 & 1.24 & 1.23 & 1.23 & 1.22 & 1.31 & 1.31 & 1.30 & 1.30 \\
$P_{P}[\%]$ & 0.06 & 0.08 & 0.08 & 0.09 & 0.08 & 0.08 & 0.08 & 0.08 & 0.08 \\
$P_{D}[\%]$ & 8.41 & 8.68 & 8.69 & 8.70 & 8.71 & 8.61 & 8.61 & 8.62 & 8.63 \\
$P_{\Delta}[\%]$ & - & 1.71 & 1.64 & 1.93 & 1.79 & 0.92 & 0.87 & 1.05 & 1.00 \\
$P_{\pi}[\%]$ & - & 0.06 & - & - & - & 0.04 & - & - & - \\
\hline \hline
\end{tabular}


The version $S$ based on soft form factors in the transition potential is tested for many observables of the two-nucleon system above pion threshold and its successes and failures are well documented in Refs. [4.5]: version $S$ is a moderatly realistic force model. In contrast, the version $H$ based on hard form factors in the transition potential has not been tested yet: its realistic nature above pion threshold is doubtful: version $H$ is used in this paper. since the original coupled-channel calculations $[9,10]$ for the trinucleon bound state were based on its transition potential. Thus, item (1) of the program list is not carried out with any satisfaction. The two versions are labelled $H(1)$ and $S(1)$ in Table I which summarizes their defining properties and in Table II which collects their predictions for the triton binding energy $E_{T}$, for the effective two-nucleon and three-nucleon contributions of the $\Delta$-isobar and the pion to the binding, i.e.. $\Delta E_{2}$ and $\Delta E_{3}$ according to the technique of Ref. [10], and for the nucleonic. $\triangle$-isobar and pionic probabilites in the triton bound state. i.e.. $P_{\mathcal{L}}$. $P_{\mathcal{\perp}}$ and $P_{\pi}$.

The effect of the explicit $\Delta$-isobar and pion degrees of freedom on the trinucleon bound state properties are for the chosen versions of the full force model well isolated. Thus, item (2) of the program list is carried out.

The results derived from the full force models $H(1)$ and $S(1)$ are compared with those of corresponding standard coupled-channel calculations without explicit pion degrees of freedom. In those coupled-channel calculations the effective $\Delta$-mass $M_{\Delta}\left(z_{\Delta}, k_{\Delta}\right)$ is taken to be constant and is equated to a stable mass $m_{\Delta}^{0}$, its standard value being $1232 \mathrm{MeV}$, the resonance position in $P_{33}$ pion-nucleon scattering. Furthermore, the pionic nucleon- $\Delta$ exchange potential becomes instantaneous. In the standard coupled-channel calculation of Ref. [18] that pionic contribution is used in a local form with hard form factors, denoted by $\pi_{H}$ in Table I. The two standard coupled-channel force models are labelled $H(4)$ and $S(4) . H(4)$ is identical with the force model A3 of Ref. [18]. Table I summarizes the defining properties of the full force model in the parametrizations $H(1)$ and $S(1)$ and three coupled-channel variants of the full force model, i.e.. $H(2), H(3), H(4)$ and $S(2), S(3), S(4)$; the variants (2) and (3) interpolate between the full force models (1) and the standard coupled-channel models (4): Variant (2) works with a mass of $1290 \mathrm{MeV}$ for the stable $\Delta$-isobar which is larger than the resonance value $1232 \mathrm{MeV}$; the effective $\Delta$-mass $M_{\Delta}\left(z_{\Delta}, k_{\Delta}\right)$ is documented in Ref. [2], it is not shown again in this paper; in bound-state problems the available energy $z_{\Delta}$ of the effective $\Delta$-mass is smaller than $m_{N}$ according to Sect. IIIB and then the effective $\Delta$-mass becomes larger than its resonance value $1232 \mathrm{MeV}$ and approaches the bare mass of $1315 \mathrm{MeV}$; thus, the value of $1290 \mathrm{MeV}$ chosen as stable $\Delta$-mass in variant (2) should approximate the effective $\Delta$-mass $M_{\Delta}\left(z_{\Delta}, k_{\Delta}\right)$ rather well; variant (2) also works with the instantaneous limit $\pi_{S}$ for the pion-exchange nucleon- $\Delta$ potential: variant (2) should reproduce the results of the full force models $H(1)$ and $S(1)$ best. Variant (3) works with a stable $\Delta$-isobar mass of $1232 \mathrm{MeV}$, the resonance value, but preserves $\pi_{S}$ for the pion-exchange nucleon- $\Delta$ potential. Table II summarizes the respective trinucleon results. It is also worth noticing at this point that in models (1), based on a dynamic $\Delta$ isobar, from the two contributions to the pionic probability in Eq. (3.16), the self-energy contribution $\delta H_{0}$ dominates over the retardation term $\delta H_{1}$, which is found to be one order of magnitude lower.

The main result of this paper - in answer to item (3) of the program list - is: The trinucleon properties derived from the full force model, defined in Fig. 2 and parametrized in Sect. III.D as $H(1)$ and $S(1)$, are well approximated by those of the corresponding standard 
coupled-channel models $H(4)$ and $S(4)$ with a stable $\Delta$-isobar. The standard coupledchannel models account for all corrections of trinucleon properties due to the explicit $\lrcorner$ isobar and pion degrees of freedom within $90 \%$. The quality of the approximation can be read of from Table II where also the results of the Paris potential. the purely nucleonic reference potential for all considered force models. are listed. The quality of the approximation even improves when the parametrization of the coupled-channel model is better tuned to the full force model as for example in variant (2). On the other hand the fact that the standard coupled-channel models $H(4)$ and $S(4)$ approximate some trinucleon properties of their corresponding full force models even more successfully than their seemingly better tuned variants (3) appears to be accidental.

As expected, the force model $H(1)$ ba ed on hard form factors in the transition potential $P_{\Delta} H_{1} P_{N}$ to nucleon- $\Delta$ states vields larger probabilities $P_{\Delta}$ and $P_{\pi}$ for the $\Delta$-isobar and the pion in the trinucleon bound state than the force model $S(1)$ does with its softer form factors. In both cases. however. the probability $P_{\pi}$ of the pionic components in the wave function is extremely small: thus, the simplifying approximation $Q H_{1} Q=0$ which neglects all interactions in the pionic sector $\mathcal{H}_{\pi}$ of the Hilbert space is well justified.

\section{CONCLUSIONS}

For the first time. this paper carries out the conceptual idea underlying the previous coupled-channel calculations of Refs. [9], [10] and [18]:

A contribution to the three-nucleon force arises from the mechanism for pion production and pion absorption: that mechanism is seen in the two-nucieon system above threshold. This paper makes the step from two-nucleon reactions without and with a pion to trinucleon properties and isolates effects related to the explicit $\Delta$-isobar and pion degrees of freedom.

Furthermore, this paper justifies the general use of coupled-channel calculations with stable $\Delta$-isobars and indicates ways for improving their simulations of the full force model.

\section{ACKNOWLEDGMENTS}

M.T.P. thanks the Theory Group at CEBAF for the kind hospitality granted during her stay there. This work was funded by the Deutsche Forschungsgemeinschaft (DFG) under contract No. Sa 247/7-2 and Sa 247/7-3, by the Deutscher Akademischer Austauschdienst (DAAD) under Contract No. 322-inida-dr, by the DOE under Grant No. DE-FG0588ER40435, and by JNICT under Contract No. PBIC/C/CEN/1094/92. The calculations for this paper were performed at Regionales Rechenzentrum für Niedersachsen (Hannover), at Continuous Electron Beam Accelerator Facility (Newport News), and at National Energy Research Supercomputer Center (Livermore). 


\section{REFERENCES}

* Present address.

[1] W. Glöckle and P. L. Sauer, Europhysics News 15, 5 (1984).

[2] H. Pöpping. P. L'. Sauer and Zhang Xi-Zhen, Nucl. Phys. A474, 557 (1987), and Erratum. Nucl. Phys. A550. 563 (1992).

[3] T.-S. H. Lee. Phys. Rev. Lett. 50. 1.571 (1983);

T.-S. H. Lee and A. Matsuyama, Phys. Rev. C 32, 516 (1985): Phys. Rev. C 36, 1459 (1987).

[4] M. T. Peña. H. Garcilazo. L. Oelfke and P. L. Sauer, Phys. Rev. C 45. 1487 (1992).

[5] A. Bulla and P. L. Sauer. Few-Body Syst. 12. 141 (1992).

[6] H. Garcilazo and T. Mizutani, $\pi$ NN Systems. World Scientific, Singapore, 1990.

[7] T.-S. H. Lee and K. Ohta, Phys. Rev. C 25, 3043 (1982).

[8] P. U. Sauer, Prog. Nucl. Part. Phys. 16, 35 (1986).

[9] Ch. Hajduk and P. L. Sauer. Nucl. Phys A322, 329 (1979).

[10] Ch. Hajduk. P. L. Sauer and W. Strueve, Nucl. Phys. A405, 581 (1983).

[11] A. M. Green. Rep. Prog. Phys. 39, 1109 (1976).

[12] B. H. J. Mckellar. in Few Body Dynamics, ed. A. N. Mitra, I. Slaus, V. S. Bhasin and V. Y. Gupta (North Holland, Amsterdam, 1976) p. 508.

[13] A. Stadler and P. U. Sauer, Pliys. Rev. C 46, 64 (1992).

[14] K. Dreissigacker, S. Furui, Ch. Hajduk, P. U. Sauer and R. Machleidt, Nucl. Phys. A375, 334 (1982).

[15] P. U. Sauer, Nucl. Phys. A543, 291c (1992).

[16] M. Lacombe, B. Loiseau. J. M. Richard, R. Vinh Mau, P. Pirès and R. de Tourreil. Phys. Rev. D 12. 1495 (1975).

[17] A. Stadler. W. Glöckle and P. L. Sauer. Phys. Rev. C 44, 2319 (1991).

[18] . M. T. Peña. H. Henning and P. L. Sauer, Phys. Rev. C 42, 855 (1990).

[19] M. Lacombe, B. Loiseau. J. M. Richard, R. Vinh Mau, J. Côté, P. Pirès and R. de Tourreil, Phys. Rev. C 21. 861 (1980).

[20] A. Valcarce, F. Fernández, H. Garcilazo, M. T. Pẽna and P. U. Sauer, HannoverSalamanca Preprint, 1992.

[21] M. Stingl and A.T. Stelbovics, J. Phys. G 4, 1371 (1978); J. Phys. G 4, 1389 (1978); Nucl. Phys. A294, 391 (1978).

\section{APPENDIX A: CALCULATION OF THE EFFECTIVE SINGULAR THREE-BARYON FORCE ARISING FROM PROCESSES OF THE TYPE FIG. 4(E)}

The process of Fig. 4(e) is redrawn in Fig. 8; a characteristic process of higher order in potentials is also shown there. 


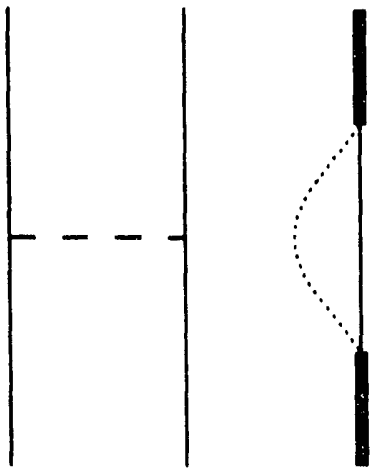

(a)

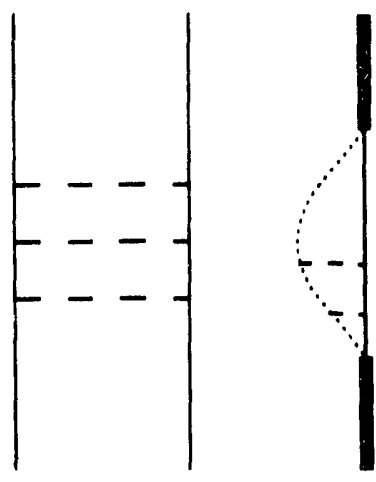

(b)

FIG. 8. Characteristic contributions to the effective three-nucleon force of the type to be calculated in this appendix. The process of Fig. $4(\mathrm{e})$ is redrawn; a characteristic process of higher order in the potentials, i.e., of third order in the two-nucleon potential and of second order in the pion-nucleon potential, is also shown.

Characteristic for both processes is that they are unlinked in a particular way: The two nucleons unconnected with the $\Delta$-isobar can interact up to infinite order of the twonucleon potential; the nucleon produced by the decay of the $\Delta$-isobar interacts with the simultaneously created pion also up to infinite order in the pion-nucleon potential, however. it does not interact with either of the other two nucleons. The processes of Fig. 8 depend on the coordinates of all three baryons. They therefore yield irreducible contributions to the effective three-baryon force. However, due to that particular disconnectedness those contributions are singular in the same way as a two-baryon interaction is singular in a threebaryon Hilbert space. The disconnectedness problem, which the process of Fig. 4(e) yields resembles the one encountered in two-nucleon scattering within the framework of $\pi . V . V$ dynamics by Ref. [21]. This paper chooses $Q H_{1} Q=0$, thus, that singular three-baryon force does not arise in the actual calculation. Nevertheless, its functional form is given in this appendix for conceptual completeness.

The interaction $Q H_{1} Q$ in the Hilbert sector $\mathcal{H}_{\pi}$ with a pion is decomposed as follows, i.e.,

$$
Q H_{1} Q==\sum_{i} v_{i}^{N N}+\sum_{i} v_{i}^{\pi N}
$$

In the two-nucleon potential $v_{i}^{N N}$ the subscript $i$ denotes the spectating nucleon, in the pion-nucleon potential $v_{i}^{\pi N}$ the subscript $i$ denotes the nucleon interacting with the pion. All potentials in the interaction $Q H_{1} Q$ are instantaneous. Processes up to infinite order in the potentials contribute; the potentials are resummed into transition matrices, i.e.,

$$
\begin{aligned}
& t_{1}^{N N}\left(z-h_{0}(1)-h_{0}(\pi)-\frac{\left(\mathbf{k}_{N 2}+\mathbf{k}_{N 3}\right)^{2}}{4 m_{N}}\right) \\
& \quad=v_{1}^{N N}\left[1+\frac{1}{\left[z-h_{0}(1)-h_{0}(\pi)-\frac{\left(\mathbf{k}_{N 2}+\mathbf{k}_{N 3}\right)^{2}}{4 m_{N}}\right]-\left(h_{0}(2)+h_{0}(3)-\frac{\left(\mathbf{k}_{N 2}+\mathbf{k}_{N 3}\right)^{2}}{4 m_{N}}\right)}\right.
\end{aligned}
$$




$$
\begin{gathered}
\left.\times t_{1}^{N N}\left(z-h_{0}(1)-h_{0}(\pi)-\frac{\left(\mathrm{k}_{N 2}+\mathrm{k}_{N 3}\right)^{2}}{4 m_{N}}\right)\right] \\
t_{1}^{\pi N}\left(z-h_{0}(2)-h_{0}(3)\right) \\
=v_{1}^{\pi N}\left[1+\frac{1}{\left[z-h_{0}(2)-h_{0}(3)\right]-h_{0}(1)-h_{0}(\pi)} t_{1}^{\pi N}\left(z-h_{0}(2)-h_{0}(3)\right)\right] .
\end{gathered}
$$

The two-nucleon transition matrix $t_{1}^{N . V}$ is defined in the c.m. system of nucleons 2 and 3; in Eq. (A2a) the pion-nucleon kinetic energy will be approximately split in the form $h_{0}(1)+h_{0}(\pi)=h_{0}^{\pi N}(1)+k_{\Delta}^{2} / 2 m_{\Delta}^{0}$ as in the context of Eq. (3.10). In contrast the pion-nucleon transition matrix $t_{1}^{\pi . N}$ is defined for a moving pion-rucleon system as for the transition matrix in Eq. (3.7): however different symbols are used for the pion-nucleon transition matrices of Eqs. (A2b) and (3.T). since their dynamic content is different.

According to Eq. (2.9a) only the part $W_{1}$ of the considered three-baryon force is needed for determining the Faddeev amplitude $P\left|\psi_{1}\right\rangle_{\text {: that }}$ part is calculated in the chosen basis of the Hilbert sector $\mathcal{H}_{\Delta}$. However, the matrix elements ${ }_{1}\left\langle p_{N}^{\prime} q_{N}^{\prime} \nu_{N}^{\prime}\left|W_{1}(z)\right| p_{N} q_{N} \nu_{N}\right\rangle_{1}$ and the nondiagonal matrix elements ${ }_{1}\left\langle p_{\Delta} q_{\Delta} \nu_{\Delta}\left|W_{1}(z)\right| p_{N} q_{N} \nu_{N}\right\rangle_{1}$ are ideititically zero. only the matrix elements for the basis states $\left|p_{\Delta} q_{\Delta} \nu_{\Delta}\right\rangle_{1}$ are nonzero and have to be computed. The operator $W_{1}$ of the considered three-baryon force is build up by simpler quantities, i.e., the twu-nucleon and pion-nucleon transition matrizes $t_{1}^{N N}$ and $t_{1}^{\pi N}$. Those transition matrices act in the Hilbert sector $\mathcal{H}_{\pi}$, they do not act on the three-baryon basis states $\left|p_{\Delta} q_{\Delta} \nu_{\Delta}\right\rangle_{1}$, but these outside basis states simplify those transition matrices inside $W_{1}$ by allowing the replacement of some operators through their corresponding eigenvalues, e.g.,

$$
\begin{gathered}
t_{1}^{N N}\left(z-h_{0}(1)-h_{0}(\pi)-\frac{\left(\mathbf{k}_{N 2}+\mathbf{k}_{N 3}\right)^{2}}{4 m_{N}}\right) \ldots\left|p_{\Delta} q_{\Delta} \nu_{\Delta}\right\rangle_{1} \\
=t^{N N}\left(z-h_{0 \text { rel }}^{\pi N}(1)-\frac{q_{\Delta}^{2}}{2}\left(\frac{1}{2 m_{N}}+\frac{1}{m_{\Delta}^{0}}\right)\right) \ldots\left|p_{\Delta} q_{\Delta} \nu_{\Delta}\right\rangle_{1}, \\
t_{1}^{\pi N}\left(z-h_{0}(2)-h_{0}(3)\right) \ldots\left|p_{\Delta} q_{\Delta} \nu_{\Delta}\right\rangle_{1}=t^{\pi N}\left(z-2 m_{N}-\frac{p_{\Delta}^{2}}{m_{N}}-\frac{q_{\Delta}^{2}}{4 m_{N}}, q_{\Delta}\right) \ldots\left|p_{\Delta} q_{\Delta} \nu_{\Delta}\right\rangle_{1} .
\end{gathered}
$$

The dots in both equations indicate that the transition matrices do not act directly on the basis states $\left|p_{\Delta} q_{\Delta} \nu_{\Delta}\right\rangle_{1}$. Both transition matrices remain operators with respect to the relative pion-nucleon motion. 

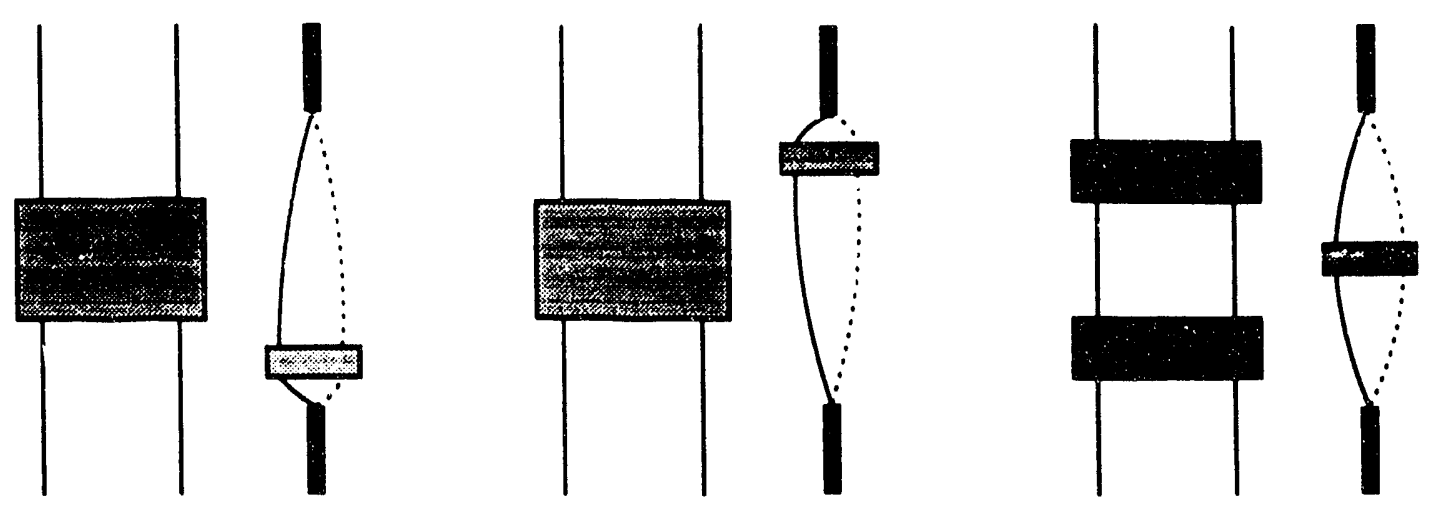

FIG. 9. The three contributions of first order in the nonresonant pion-nucleon transition matrix to the effective three-nucleon force. calculated in this appendix. The shaded boxes denote nucleon-nucleon and pion-nucleon transition matrices. they can be differentiated by their external legs.

The pion-nucleon transition matrix $t^{\pi \cdot \nu}$ of Eq. (A3b) sums up the nonresonant part of the pion-nucleon interaction in the $P_{33}$ partial wave. The nonresonant part is weak. Thus, only contributions of first order in the pion-nucleon transition matrix $t^{\pi N}$ are considered. The three arising contributions are shown in Fig. 9. They have the following analytic form

$$
\begin{aligned}
& { }_{1}\left\langle p_{\Delta}^{\prime} q_{\Delta}^{\prime} \nu_{\Delta}^{\prime}\left|W_{1}\right| p_{\Delta} q_{\Delta} \nu_{\Delta}\right\rangle_{1}= \\
& \frac{\delta\left(q_{\Delta}^{\prime}-q_{\Delta}\right)}{q_{\Delta}^{2}}\langle f| \frac{1}{\left[z-2 m_{N}-\frac{p_{\Delta}^{\prime 2}}{m_{. V}}-\frac{z_{\Delta}^{2}}{2}\left(\frac{1}{2 m_{.:}}+\frac{1}{m_{\Delta}^{0}}\right)\right]-h_{0 \text { rel }}^{\pi N}(1)} \\
& \times\left\{p_{\Delta}^{\prime} \nu_{\Delta}^{\prime}: t^{\cdots} \nu\left(z-\frac{q_{\Delta}^{2}}{2}\left(\frac{1}{2 m_{N}}+\frac{1}{m_{\Delta}^{0}}\right)-h_{0 \text { rel }}^{r \cdot v}(1)\right)\left|p_{\Delta} \nu_{\Delta}\right\rangle\right. \\
& \times \frac{1}{\left[z-2 m_{N}-\frac{p_{\Delta}^{2}}{m_{N}}-\frac{q_{\Delta}^{2}}{2}\left(\frac{1}{2 m_{N}}+\frac{1}{m_{\Delta}^{\sigma}}\right)\right]-h_{0 \text { rel }}^{\pi N}(1)} \\
& \times t^{\pi N}\left(z-2 m_{N}-\frac{p_{\Delta}^{2}}{m_{N}}-\frac{q_{\Delta}^{2}}{2}\left(\frac{1}{2 m_{N}}+\frac{1}{m_{\Delta}^{0}}\right), q_{\Delta}\right) \\
& +\sum_{\nu_{\Delta}^{\prime \prime}} \int p_{\Delta}^{\prime \prime 2} d p_{\Delta}^{\prime \prime}\left\langle p_{\Delta}^{\prime} \nu_{\Delta}^{\prime}\left|t^{N N}\left(z-\frac{q_{\Delta}^{2}}{2}\left(\frac{1}{2 m_{v}}+\frac{1}{m_{\Delta}^{0}}\right)-h_{0 \mathrm{rel}}^{\pi N}(1)\right)\right| p_{\Delta}^{\prime \prime} \nu_{\Delta}^{\prime \prime}\right\rangle \\
& \times \frac{1}{\left[z-2 m_{N}-\frac{p_{\Delta}^{\prime \prime 2}}{m_{N}}-\frac{q_{\Delta}^{2}}{2}\left(\frac{1}{m_{N}}+\frac{1}{m_{\Delta}^{9}}\right)\right]-h_{0 \text { rel }}^{n: N}(1)} \\
& \times t^{\pi N}\left(z-2 m_{N}-\frac{p_{A}^{\prime 2}}{m_{N}}-\frac{q_{\Delta}^{2}}{2}\left(\frac{1}{2 m_{N}}+\frac{1}{m_{\Delta}^{0}}\right), q_{\Delta}\right) \\
& \times \frac{1}{\left[z-2 m_{N}-\frac{p_{\Delta}^{\prime \prime 2}}{m_{N}}-\frac{q_{\Lambda}^{2}}{2}\left(\frac{1}{2 m_{N}}+\frac{1}{m_{\Delta}^{0}}\right)\right]-h_{0 \text { rel }}^{\pi N}(1)} \\
& \times\left\langle p_{\Delta}^{\prime \prime} \nu_{\Delta}^{\prime \prime}\left|t^{N N}\left(z-\frac{q_{\Delta}^{2}}{2}\left(\frac{1}{2 m_{N}}+\frac{1}{m_{\Delta}^{\theta}}\right)-h_{0 \text { rel }}^{\pi \cdot N}(1)\right)\right| p_{\Delta} \nu_{\Delta}\right\rangle
\end{aligned}
$$




$$
\begin{aligned}
& +t^{\pi N}\left(z-2 m_{N}-\frac{p_{\Delta}^{\prime 2}}{m_{N}}-\frac{q_{\Delta}^{2}}{2}\left(\frac{1}{2 m_{. N}}+\frac{1}{m_{\Delta}^{0}}\right) \cdot q_{\Delta}\right) \\
& \times \frac{1}{\left[z-2 m_{. v}-\frac{p_{I}^{\prime 2}}{m_{. v}}-\frac{q_{\Delta}^{2}}{2}\left(\frac{1}{2 m_{. v}}+\frac{1}{m_{\Delta}^{3}}\right)\right]-h_{0}^{\pi \cdot v}(1)}
\end{aligned}
$$

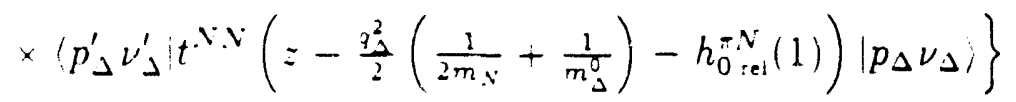

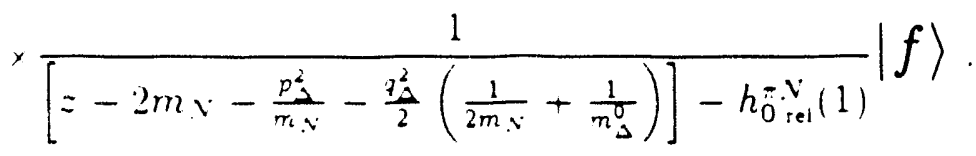

The $\delta$-function $\delta\left(q_{\Delta}^{\prime}-q_{\Delta}\right) / q_{\Delta}^{2}$ yields the singular structure of the three-baryon force $W_{1}^{\prime}(z)$.

In calculations with $Q H_{1} Q \neq 0$ the effective three-baryon force $W_{i}(z)$ arises and has the iiscussed singular part of Eq. (At). That singular part has to be corntined with the two-baryon interaction $v_{1}(z)$ of same singularity structure.

\title{
DISCLAIMER
}

\begin{abstract}
This report was prepared as an account of work sponsored by an agency of the United States Government Neither the United States Government nor any agency thereof, nor any of their empioyees, makes any warranty, express or implied, or assumes any legal liability of responsibility for the accuracy, completeness, or usefulness of any information, apparatus, product, or process disclosed, or represents that its use would not infringe privately owned rights. Reference herein to any specific commercial product, process, or service by trade name, trademark, manufacturer, or otherwise does not necessarily constitute or imply its endorsement, recommendation, or favoring by the United States Government or any agency thereof. The views and opinions of authors expressed herein do not necessarily state or reflect those of the United States Government or any agency thereof.
\end{abstract}



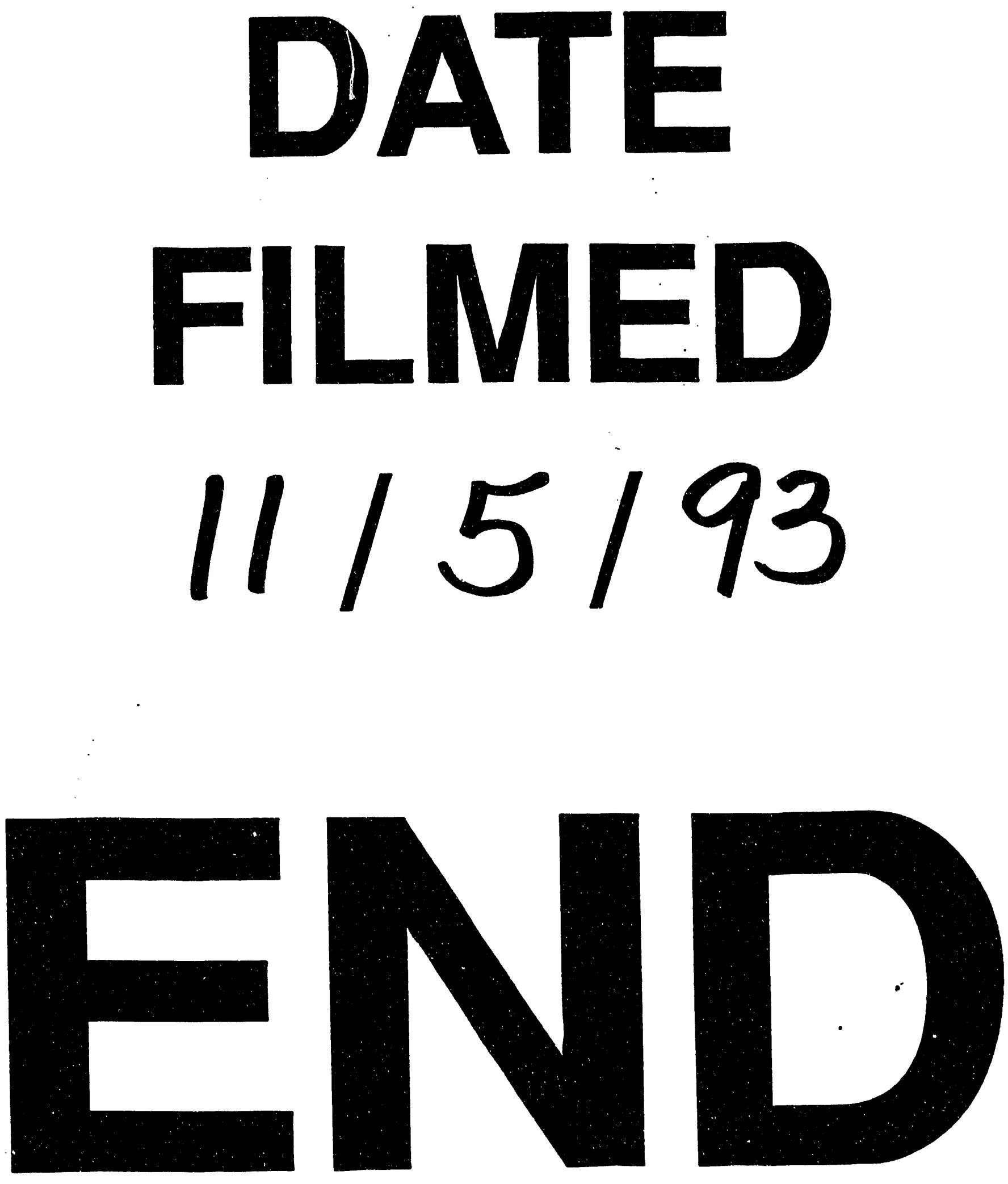
